\title{
AdS/CFT in the Infinite Momentum Frame
}

\author{
D. Brecher* \\ Centre for Particle Theory, Department of Mathematical Sciences, \\ University of Durham, South Road, Durham DH1 3LE, United Kingdom. \\ A. Chamblin ${ }^{\dagger}$ \\ Center for Theoretical Physics, Massachusetts Institute of Technology, Bldg. 6-304, \\ Cambridge, MA 02139, U.S.A. \\ H.S. Reall ${ }^{\ddagger}$ \\ Physics Department, Queen Mary College, \\ Mile End Road, London E1 4NS, United Kingdom.
}

Preprint DTP/00/103, QMW-PH/00-15, MIT-CTP-3052

8 December 2000

\begin{abstract}
This paper considers the spacetimes describing pp-waves propagating on extremal non-dilatonic branes. It is shown that an observer moving along a geodesic will experience infinite curvature at the horizon of the brane, which should therefore be regarded as singular. Taking the decoupling limit of these brane-wave spacetimes gives a pp-wave in AdS, the simplest example being the Kaigorodov spacetime. It has been conjectured that gravity in this spacetime is dual to a CFT in the infinite momentum frame with constant momentum density. If correct, this implies that the CFT must resolve the singularity of the bulk spacetime. Evidence in favour of this conjecture is presented. The unbroken conformal symmetries determine the scalar 2-point function up to an arbitrary function of one variable. However, an AdS/CFT calculation shows that this function is constant (to leading order in $1 / N^{2}$ ) and the result is therefore the same as when the full conformal symmetry is unbroken. This paper also discusses a recently discovered Virasoro symmetry of metrics describing pp-waves in AdS and naked singularities in the Randall-Sundrum scenario.
\end{abstract}

\section{Introduction}

In the standard picture 11, when one thinks of the global causal structure of non-dilatonic $p$-branes one thinks of vacuum interpolation. Far from the brane, the fields associated with the brane typically fall off quickly and the spacetime approaches uncompactified, flat space. Near the brane, the geometry is usually that of a wormhole, or Einstein-Rosen bridge. As one approaches the brane, one falls down the throat of the wormhole and approaches some vacuum which is a valid compactification of the supergravity theory. Thus, while it is often impossible to actually locate the "brane worldvolume" in these effective field theory solutions which describe gravitating branes, it is still possible to discuss the causal structure of the configurations. In particular, it is always the case that different supersymmetric

\footnotetext{
*email: Dominic.Brecher@durham.ac.uk

†email: chamblin@mit.edu

${ }^{\ddagger}$ email: H.S.Reall@qmw.ac.uk
} 
vacuum solutions of the supergravity theory emerge in different regions of the full spacetime of a $p$ brane. Furthermore, when the brane worldvolume is flat there are no curvature singularities in the near-horizon region and so we may extend the spacetime through the horizon.

A natural question is: what happens to this causal structure when one perturbs the brane? In this paper, we consider a large class of perturbations of the near-horizon region of the brane spacetime, corresponding to plane fronted parallel gravitons moving tangent to the brane directions. We find that such Weyl curvature fluctuations always lead to the appearance of pp-curvature singularities in the near-horizon region. In other words, some curvature components diverge in an orthonormal frame that is parallelly propagated along a geodesic that reaches the horizon. Thus, these perturbations close off the spacetime, so that there is no analytic extension of the manifold through the near-horizon region. Similar results have been previously noticed for waves on strings [2, 3, 4]. It has also been shown that if one attempts to extend the metric beyond the singular string horizon, then a test string falling through the horizon would experience a divergent excitation [5]. Therefore, these singularities should be regarded as a physical boundary to the spacetime.

The pp-wave in the spacetimes that we consider is described by a harmonic function which can depend on both transverse and worldvolume brane coordinates. It has been proposed that such solutions can describe fully localized intersections of a pp-wave with a brane [6], and explicit solutions for such a localized intersection have been presented in the near horizon limit [7]. We shall show that it is possible to derive the near horizon solution of [f] simply by requiring that the horizon be free of ppsingularities. However, we shall then show that the resulting solution can be related to anti-de Sitter space (AdS) by a coordinate transformation, so this solution does not really describe a wave. The only non-trivial non-singular solutions have a harmonic function that vanishes on the brane horizon. Such solutions describe a wave propagating in a direction parallel to the brane, but at some distance away from the horizon, rather than a wave on the brane worldvolume. Our results, and the results of [2, 3] are evidence in favour of a generalized "no-hair" theorem for time dependent perturbations of brane horizons.

Taking the decoupling limit [8] whilst keeping the momentum density of the wave finite, one obtains solutions describing pp-waves propagating in AdS. The case in which the harmonic function describing the wave depends only on the transverse direction was discussed in [9], where it was shown that the Kaigorodov spacetime [10, 11] is obtained in the decoupling limit. It was also shown that if the brane is made non-extremal then the wave can be removed by a boost. In the limit of extremality, this boost becomes infinite. It is therefore natural to regard the extremal brane-wave solution as an infinitely boosted version of the extremal brane solution. In the decoupling limit, this means that the Kaigorodov spacetime can be regarded as a infinitely boosted version of AdS. This infinite boost also induces an infinite boost on the conformal boundary of AdS. In the light of the AdS/CFT correspondence [8], it is therefore natural to conjecture [9] that string theory in the Kaigorodov spacetime (times a sphere of appropriate dimension) is dual to some conformal field theory (CFT) in an infinitely boosted frame, i.e., the so-called infinite momentum frame. Since the momentum density was held fixed in the decoupling limit, there must be a non-zero background momentum density present. If this conjecture is correct then the CFT must resolve the singularity of the bulk spacetime.

We have calculated the expectation value of the CFT energy-momentum tensor that arises from a large class of solutions describing pp-waves propagating along the horospheres of AdS. The energymomentum tensor describes a null momentum density, with profile fixed by the profile of the bulk wave. For the Kaigorodov spacetime, the momentum density is indeed constant, in agreement with the conjecture of [9]. This background momentum density breaks the conformal symmetry group of the boundary theory down to some smaller symmetry group. We show how the isometries of the Kaigorodov spacetime have a natural interpretation as this subgroup of the conformal group that leaves the background momentum density invariant. 
When conformal invariance is unbroken, it is well-known that the 2-point functions of certain CFT operators are completely determined. In our case, although some of the conformal invariance is broken, the dilatation symmetry persists (when combined with a boost). This highly, but not entirely, constrains the form of the 2-point functions. For a spin-0 operator, we show that the symmetry determines the 2-point function up to an arbitrary function of one variable. It is then natural to ask whether we can calculate the 2-point function using the prescription of [12, 13]. This turns out to be straightforward, with the exception that the Kaigorodov spacetime is non-static and therefore cannot be Wick rotated to Euclidean signature. The AdS/CFT calculation must therefore be carried out on the Lorentzian spacetime.

The 2-point function is not uniquely defined in Lorentzian signature. However, the arbitrariness takes a form that is characteristic of the infinite momentum frame, offering further support in favour of the conjecture of [9]. Moreover, the 2-point function turns out to be independent of the background momentum density. We argue that this makes sense in the large $N$ limit.

Another topic addressed in this paper is the Virasoro symmetry group of anti-de Sitter space. It is well-known that gravity in spacetimes asymptotic to $\mathrm{AdS}_{3}$ is equivalent to a two-dimensional CFT [14]. It has recently been pointed out that the space of metrics describing pp-waves propagating on the horospheres of AdS admits an action of the Virasoro group in any dimension [15]. We explain how this symmetry arises from the action of the Virasoro group on 3-dimensional submanifolds of these spacetimes.

\section{Singular brane-waves}

Our starting point is the ansatz for the metric of a non-dilatonic $p$-brane with a pp-wave traveling along a worldvolume direction, which we take to be $x$. The line element can be written as:

$$
d s^{2}=H(r)^{-2 / d}\left(2 d x^{+} d x^{-}+F\left(x^{+}, x^{i}, r\right) d x^{+2}+d x^{i} d x^{i}\right)+H(r)^{2 / \tilde{d}}\left(d r^{2}+r^{2} d \Omega_{\tilde{d}+1}^{2}\right),
$$

where $d=p+1, \tilde{d}=D-d-2, D$ is the dimension of spacetime and

$$
\frac{2}{d}+\frac{2}{\tilde{d}}=1
$$

for the branes of interest. $x^{i}, i=1, \ldots, d-2$, denote the directions tangent to the brane and transverse to the wave, and we have written

$$
x^{ \pm}=\frac{(x \pm t)}{\sqrt{2}} .
$$

$d \Omega_{\tilde{d}+1}^{2}$ is the line-element of a unit $(\tilde{d}+1)$-sphere (or, more generally, any positive Einstein space with appropriately normalized curvature). Where necessary, we will take $m, n=1, \ldots, \tilde{d}+1$ to denote directions in this space. The function $F\left(x^{+}, x^{i}, r\right)$ depends on both worldvolume and transverse coordinates so the wave is potentially fully localized on both the brane and in the transverse space.

The curvature tensors for this metric are calculated in the appendix. For the M2-brane, the four form field strength is

$$
F_{[4]}=d\left(H^{-1}\right) \wedge d x^{+} \wedge d x^{-} \wedge d y,
$$

where we have written $x^{1}=y$. For the M5-brane, the dual of the four form field strength is

$$
* F_{[4]}=d\left(H^{-1}\right) \wedge d x^{+} \wedge d x^{-} \wedge d x^{1} \wedge d x^{2} \wedge d x^{3} \wedge d x^{4},
$$


and for the D3-brane, the self-dual five form field strength is

$$
F_{[5]}=G_{[5]}+* G_{[5]}
$$

where

$$
G_{[5]}=d\left(H^{-1}\right) \wedge d x^{+} \wedge d x^{-} \wedge d x^{1} \wedge d x^{2} .
$$

It is straightforward to verify that these expressions solve the Einstein equations provided that the function $H(r)$ is harmonic on the transverse space:

$$
H(r)=1+\frac{k}{r^{\tilde{d}}},
$$

and the function $F\left(x^{+}, x^{i}, r\right)$ obeys (e.g., [16, 6, , 7])

$$
\frac{\partial^{2} F}{\partial r^{2}}+\frac{(\tilde{d}+1)}{r} \frac{\partial F}{\partial r}+H(r) \nabla_{\perp}^{2} F=0
$$

where $\nabla_{\perp}^{2}$ denotes the Laplacian with respect to the flat coordinates $x^{i}$.

There are different types of solution to this equation, but we will not consider the specific forms here. Suffice it to say that the usual brane-wave intersections [16] have $F=F\left(x^{+}, r\right)$, the wave being localized in the transverse space only. On the other hand, a Ricci-flat brane [17, 18, 19] can be constructed by taking $F=F\left(x^{+}, x^{i}\right)$, in which case the wave is now localized on the brane worldvolume, but not in the transverse space. In the near-horizon limit, one obtains solutions describing pp-waves propagating in anti-de Sitter space. These were discussed in four dimensions in [20]. They have also been used to give a non-linear discussion of the Randall-Sundrum [21] model, with the solution $F=H_{i j}\left(x^{+}\right) x^{i} x^{j}$, (where $H_{i j}$ is symmetric and trace-free) identified as the massless graviton [22].

There is a particular solution which will prove of interest in what follows. In the core of the brane, as $r \rightarrow 0$, (2.9) becomes

$$
\frac{\partial^{2} F}{\partial r^{2}}+\frac{(\tilde{d}+1)}{r} \frac{\partial F}{\partial r}+\frac{k}{r^{\tilde{d}}} \nabla_{\perp}^{2} F=0
$$

which is solved by [7, 25]

$$
F\left(x^{+}, x^{i}, r\right)=F_{0}\left(x^{+}\right)+F_{1}\left(x^{+}\right)\left[|x|^{2}+k\left(\frac{d-2}{\tilde{d}-2}\right) \frac{1}{r^{\tilde{d}-2}}\right] .
$$

This solution can, in fact, be extended beyond the near-horizon region. By writing $F=F_{0}\left(x^{+}\right)+$ $F_{1}\left(x^{+}\right)\left(|x|^{2}+f(r)\right)$, we find that

$$
F\left(x^{+}, x^{i}, r\right)=F_{0}\left(x^{+}\right)+F_{1}\left(x^{+}\right)\left[|x|^{2}-\left(\frac{d-2}{\tilde{d}+2}\right) r^{2}+k\left(\frac{d-2}{\tilde{d}-2}\right) \frac{1}{r^{\tilde{d}-2}}\right],
$$

is a solution of (2.9), valid for all $r$. However, the amplitude of the wave grows as one moves away from the brane, and destroys the asymptotic flatness of the spacetime. It is therefore unlikely that this solution is of physical relevance. 


\section{$2.1 \quad$ Near-horizon geometry}

In the near horizon limit of the metric (2.1), the 1 in the harmonic function $H$ can be dropped. Defining a new coordinate by

$$
z=\frac{d}{\tilde{d}} k^{1 / 2} r^{1-\tilde{d} / 2}
$$

then brings the metric to the form

$$
d s^{2}=\frac{l^{2}}{z^{2}}\left(2 d x^{+} d x^{-}+F\left(x^{+}, x^{i}, z\right) d x^{+^{2}}+d x^{i} d x^{i}+d z^{2}\right)+(\tilde{d} / d)^{2} l^{2} d \Omega_{\tilde{d}+1}^{2}
$$

where

$$
l=(d / \tilde{d}) k^{1 / \tilde{d}} .
$$

In the new coordinate, the equation satisfied by $F$ is

$$
z^{d-1} \frac{\partial}{\partial z}\left(\frac{1}{z^{d-1}} \frac{\partial F}{\partial z}\right)+\nabla_{\perp}^{2} F=0
$$

Throwing away the $d \Omega_{\tilde{d}+1}^{2}$ piece of the spacetime (2.14), and with the equation (2.16) governing the function $F$, we recognise the metric describing a pp-wave in $\operatorname{AdS}_{d+1}$. The AdS horizon is now at $z=\infty$, and the conformal boundary at $z=0$. The latter is, in general, no longer Minkowski space; and we will have more to say on this below.

\subsection{Supersymmetry considerations}

It is easy enough to see that our brane-waves preserve $1 / 4$ of the spacetime supersymmetries, as should be the case for a two-element intersection. In the AdS limit considered above, however, one might expect a supersymmetry enhancement. It is perhaps surprising that this does not occur. Indeed, the Kaigorodov spacetime preserves $1 / 4$ of the supersymmetries [9] and, as we will now show, this is true of any pp-wave in AdS.

Consider, then, the spacetime (2.14) without the $d \Omega_{\tilde{d}+1}^{2}$ piece. The Killing spinor equations for a $(d+1)$-dimensional supergravity theory with cosmological constant $\Lambda=-\left(1 / 2 l^{2}\right) d(d-1)$ have the form

$$
\delta \psi_{M} \equiv \mathcal{D}_{M} \epsilon=D_{M} \epsilon-\frac{1}{2 l} \Gamma_{M} \epsilon=0,
$$

where $D_{M}$ is the covariant derivative acting on an arbitrary spinor $\epsilon$. It is useful to consider the integrability condition for the existence of Killing spinors, which is

$$
H_{M N} \epsilon \equiv\left[\mathcal{D}_{M}, \mathcal{D}_{N}\right] \epsilon=\frac{1}{4} R_{M N P Q} \Gamma^{P Q} \epsilon+\frac{1}{2 l^{2}} \Gamma_{M N} \epsilon=0 .
$$

Substituting for the Riemann tensor, we find the non-zero (tangent space) components of this equation to be

$$
\begin{gathered}
H_{+i} \epsilon=-\frac{1}{2} \frac{z^{2}}{l^{2}}\left[\left(\frac{\partial^{2} F}{\partial x^{i} \partial x^{j}}-\frac{1}{z} \frac{\partial F}{\partial z} \delta_{i j}\right) \Gamma_{j}-\frac{\partial^{2} F}{\partial z \partial x^{i}} \Gamma_{z}\right] \Gamma_{-} \epsilon=0, \\
H_{+z} \epsilon=-\frac{1}{4} \frac{z^{2}}{l^{2}}\left[\left(\frac{\partial^{2} F}{\partial z^{2}}-\frac{1}{z} \frac{\partial F}{\partial z}\right) \Gamma_{z}-\frac{\partial^{2} F}{\partial z \partial x^{i}} \Gamma_{i}\right] \Gamma_{-} \epsilon=0,
\end{gathered}
$$

where all gamma matrices are defined with respect to the tangent space metric. 
The conditions (2.19,2.20) can be satisfied in one of two ways. The first is to take

$$
\frac{\partial^{2} F}{\partial z \partial x^{i}}=\frac{\partial^{2} F}{\partial z^{2}}-\frac{1}{z} \frac{\partial F}{\partial z}=\frac{\partial^{2} F}{\partial x^{i} \partial x^{j}}-\frac{1}{z} \frac{\partial F}{\partial z}=0,
$$

which is solved by

$$
F\left(x^{+}, x^{i}, z\right)=F_{0}\left(x^{+}\right)+F_{1}\left(x^{+}\right)\left[|x|^{2}+z^{2}\right] .
$$

This is just the solution (2.11) in the $z$ coordinate. However, as we shall show below, it in fact corresponds to pure AdS in an unusual coordinate system, for which the integrability equations are satisfied identically; for bona fide waves, the function $F$ cannot have this form. So we are led to the second way of satisfying the integrability conditions (2.19,2.20), which is of course to demand that

$$
\Gamma_{-} \epsilon=0 .
$$

This is just the usual chirality condition for a pp-wave to be supersymmetric, and removes $1 / 2$ of the supersymmetries. However, since this is a necessary, but not sufficient, condition to ensure the existence of Killing spinors, we must further consider the equations (2.17) explicitly.

The Killing spinor equations (2.17) reduce to the following conditions on $\epsilon$ :

$$
\begin{gathered}
\partial_{+} \epsilon=\frac{1}{2 z} \Gamma_{+}\left(1+\Gamma_{z}\right) \epsilon+\frac{1}{4}\left[\frac{\partial F}{\partial x^{i}} \Gamma_{i}+\left(\frac{\partial F}{\partial z}-\frac{F}{z}\right) \Gamma_{z}\right] \Gamma_{-} \epsilon \\
\partial_{-} \epsilon=\frac{1}{4 z}\left(1-\Gamma_{z}\right) \Gamma_{-} \epsilon \\
\partial_{i} \epsilon=\frac{1}{2 z} \Gamma_{i}\left(1+\Gamma_{z}\right) \epsilon \\
\partial_{z} \epsilon=\frac{1}{2 z} \Gamma_{z} \epsilon .
\end{gathered}
$$

Solutions of these equations must satisfy the chirality condition (2.23). This removes all trace of the function $F$, leaving the pure AdS equations, for which the Killing spinors were constructed in horospherical coordinates in [26]. In this case, there are two distinct types of solution: one a function of all the coordinates, and one a function of $z$ alone. Only the latter type of solution satisfies equation (2.23), however. In other words, the sole solution to the Killing spinor equations is

$$
\epsilon=\sqrt{\frac{l}{z}} \epsilon_{0}
$$

where $\epsilon_{0}$ is a constant spinor satisfying

$$
\begin{aligned}
\left(1+\Gamma_{z}\right) \epsilon_{0} & =0, \\
\Gamma_{-} \epsilon_{0} & =0,
\end{aligned}
$$

The pp-wave in AdS thus preserves only 1/4 of the supersymmetries. It is important to note that this is true regardless of which coordinates $F$ is a function of. In particular, it is true of the Kaigorodov spacetime, to be considered below, as shown in [9]. At any rate, the pp-wave in $\mathrm{AdS}_{5}$ should break the supersymmetry of the dual CFT from $\mathcal{N}=4$ to $\mathcal{N}=1$. This can occur in two different ways: either the pp-wave changes the background metric of the boundary theory, which explicitly breaks some of the supersymmetry, or the pp-wave preserves the flat metric of the boundary theory but corresponds to a quantum state of the boundary theory in which some of the supersymmetry is spontaneously broken. In the case of the Kaigorodov metric, the latter possibility occurs, with the symmetry breaking arising from a non-zero expectation value for the energy-momentum tensor. 


\subsection{Global structure}

Since the pp-wave generates Weyl curvature alone, the only potentially divergent curvature invariant of our solutions is the square of the Riemann tensor; and it is easy to see from the curvature components given in the appendix that this is everywhere finite. However, it was shown in [20] that pp-waves in $\mathrm{AdS}_{4}$ generically give rise to pp-curvature singularities at the AdS horizon, i.e., in an orthonormal frame parallelly propagated along a causal geodesic, some curvature components will diverge at the AdS horizon. It is therefore important that we examine the behaviour of the tidal forces experienced by observers who move along geodesics in brane-wave spacetimes.

Consider, then, the geodesic equations for the spacetime (2.1). For simplicity, we take $d \Omega_{\tilde{d}+1}^{2}$ to be the line-element on the unit $(\tilde{d}+1)$-dimensional sphere, set all the polar angles to $\pi / 2$, and take $\phi$ to be the azimuthal angle. We further take $t^{M}$ to denote the unit tangent to a timelike geodesic, with affine parameter $\lambda$. Then the Killing vectors $k=\frac{\partial}{\partial x^{-}}$and $m=\frac{\partial}{\partial \phi}$ generate the conserved quantities $E=k \cdot t$, and $L=m \cdot t$ respectively. These give the geodesic equations

$$
\begin{aligned}
\frac{d x^{+}}{d \lambda} & =E H^{2 / d} \\
\frac{d \phi}{d \lambda} & =\frac{L}{r^{2} H^{2 / \tilde{d}}} .
\end{aligned}
$$

The $x^{i}$ equation is

$$
\frac{d}{d \lambda}\left(\frac{1}{H^{2 / d}} \frac{d x^{i}}{d \lambda}\right)=\frac{E^{2}}{2} H^{2 / d} \frac{\partial F}{\partial x^{i}}
$$

and that governing the radial motion is

$$
\begin{aligned}
\frac{d}{d \lambda}\left(H^{2 / \tilde{d}} \frac{d r}{d \lambda}\right)= & \frac{1}{2} \frac{H^{\prime}}{H}\left(\frac{2}{d}+H^{2 / \tilde{d}}\left(\frac{d r}{d \lambda}\right)^{2}\right)+\frac{1}{2} E^{2} H^{2 / d} \frac{\partial F}{\partial r} \\
& +\frac{1}{2} \frac{L^{2}}{r^{2} H^{2 / \tilde{d}}}\left(\frac{2}{r}+\frac{H^{\prime}}{H}\right),
\end{aligned}
$$

For the special case in which $F=F\left(x^{+}, x^{i}\right)$ and $L=0$, this equation can be solved to give

$$
\frac{d r}{d \lambda}=\frac{1}{H^{(d-2) / 2 d}} \sqrt{c H^{2 / d}-1}
$$

for some constant $c$. In general, however, we are unable to solve $(2.34)$ due to the $r$-dependence of the function $F$. Using the timelike condition, the final equation for $x^{-}$is

$$
\frac{d x^{-}}{d \lambda}=-\frac{1}{2 E}\left(1+\frac{1}{H^{2 / d}}\left(\frac{d x^{i}}{d \lambda}\right)^{2}+H^{2 / \tilde{d}}\left(\frac{d r}{d \lambda}\right)^{2}+E^{2} H^{2 / d} F+\frac{L^{2}}{r^{2} H^{2 / \tilde{d}}}\right) .
$$

As we have already mentioned, we cannot solve this set of equations in general. But this does not prevent us from analyzing the near-horizon geometry of the brane. To this end, we set $L=0$ and write the tangent vector to the geodesic as

$$
t^{M}=\left(E H^{2 / d}, \dot{x}^{-}, \dot{\mathbf{x}}, \dot{r}, 0, \ldots, 0\right),
$$

where $\dot{x}^{+}=E H^{2 / d}$, a dot denotes differentiation with respect to $\lambda, \mathbf{x}=\left\{x^{i}\right\}$ and we have written the components in the order $\left(t^{+}, t^{-}, t^{i}, t^{r}, t^{\theta_{1}}, \ldots, t^{\theta} \tilde{d}, t^{\phi}\right)$. We want to analyze the behaviour of the 
components of the Riemann tensor in an orthonormal frame parallelly propagated along a geodesic with tangent $t^{M}$. We must first construct the other vectors of this orthonormal frame. There are $d-2$ unit normals of the form

$$
n_{i}^{M}=H^{1 / d}\left(0,-\frac{\dot{x}^{i}}{E H^{2 / d}}, \hat{n}_{i}, 0, \ldots, 0\right)
$$

where $\hat{n}_{i}^{j}=\delta_{i}^{j}$. The normals satisfy $t \cdot n_{i}=0, n_{i} \cdot n_{j}=\delta_{i j}$ and it is easy to check that they are parallelly propagated with respect to $t$ :

$$
t \cdot \nabla n_{i}^{M}=0 .
$$

Although they will prove to be irrelevant to our discussion, the $\tilde{d}+1$ angular normals, $n_{m}$, are easy to determine. They have a single non-zero component

$$
n_{m}^{n}=\frac{1}{H^{1 / \tilde{d} r}} \frac{1}{\sin \theta_{1} \ldots \sin \theta_{\tilde{d}}} \delta_{m}^{n} .
$$

The remaining two normals of our parallelly propagated frame are somewhat harder to determine. Denoting them by $p$ and $q$, we have

$$
\begin{aligned}
& p^{M}=\sin \mu(\lambda)\left(E H^{2 / d}, \dot{x}^{-}+\frac{1}{E}, \dot{\mathbf{x}}, \dot{r}, 0, \ldots, 0\right)+\cos \mu(\lambda)\left(0, \frac{\dot{r}}{E} H^{1 / \tilde{d}}, \mathbf{0},-H^{-1 / \tilde{d}}, 0, \ldots, 0\right), \\
& q^{M}=\cos \mu(\lambda)\left(E H^{2 / d}, \dot{x}^{-}+\frac{1}{E}, \dot{\mathbf{x}}, \dot{r}, 0, \ldots, 0\right)-\sin \mu(\lambda)\left(0, \frac{\dot{r}}{E} H^{1 / \tilde{d}}, \mathbf{0},-H^{-1 / \tilde{d}}, 0, \ldots, 0\right),
\end{aligned}
$$

where $\mu(\lambda)$ obeys

$$
\frac{d \mu}{d \lambda}=-\frac{H^{\prime}}{d H} H^{-1 / \tilde{d}}
$$

which gives $\mu=$ constant far from the brane $(r \rightarrow \infty)$, and $\mu=\lambda / l+$ constant in the near horizon region $(r \rightarrow 0)$. These normals are slight generalizations of those given by Podolský [20] in a study of pp-waves in $\mathrm{AdS}_{4}$.

\subsection{Waves in $\mathbf{A d S}_{d+1}$}

Before analyzing the global structure of our brane-waves, it will be instructive to consider the simpler case of waves in $\mathrm{AdS}_{d+1} \times S^{\tilde{d}+1}$, the near-horizon geometry of the metric (2.1). Note that it is not sufficient to just consider waves in AdS when studying pp-singularities at the horizon of the brane-wave spacetime. This is because in taking the near horizon limit, one has to take the limit of the components of the curvature in a parallelly propagated frame, e.g., of objects like $t^{M} p^{N} t^{P} p^{Q} R_{M N P Q}$. However, in the AdS limit, one takes the limits of $t^{M}, p^{N}$ and $R_{M N P Q}$ separately and then multiplies them. As we shall see, the product of these limits is not necessarily equal to the limit of the product. Therefore it is necessary to examine the brane-wave spacetime and the pp-wave in AdS separately.

The presence of the sphere is irrelevant, so we will essentially be considering waves in $\mathrm{AdS}_{d+1}$, and the results are again a slight generalization of those in [20]. To look at the AdS case, as opposed to our brane-wave spacetime (2.1), we can simply set $H(r)=k / r^{\tilde{d}}$ in the expressions for the curvature components, tangent vector and normals. In this case the equation of motion (2.10) valid in the core of the brane, is exact. Analysis of the curvature components in our parallelly propagated frame is then fairly straightforward. 
There are a number of potential pp-singularities, but they are all encoded in the quantities円

$$
\begin{aligned}
\mathcal{A}_{+} & =\frac{E^{2}}{2}\left(\frac{k}{r \tilde{d}}\right)^{1-4 / \tilde{d}}\left[F^{\prime \prime}+(\tilde{d}-1) \frac{F^{\prime}}{r}\right], \\
\mathcal{A}_{i} & =\frac{E^{2}}{2}\left(\frac{k}{r \tilde{d}}\right)^{3 / 2-4 / \tilde{d}} \frac{\partial^{2} F}{\partial r \partial x^{i}}, \\
\mathcal{M}_{i j} & =\frac{E^{2}}{2}\left(\frac{k}{r \tilde{d}}\right)^{2-4 / \tilde{d}}\left[\frac{\partial^{2} F}{\partial x^{i} \partial x^{j}}+\delta_{i j} \frac{\tilde{d}}{d} \frac{r^{\tilde{d}}}{k} \frac{F^{\prime}}{r}\right],
\end{aligned}
$$

which are potentially divergent near the AdS horizon $(r \rightarrow 0)$. All components of the curvature tensor in our parallelly propagated frame depend on these three quantities alone. For example,

$$
\begin{gathered}
R_{(t)(p)(t)(p)} \equiv R_{M N P Q} t^{M} p^{N} t^{P} p^{Q}=\frac{1}{l^{2}}-\mathcal{A}_{+} \cos ^{2}(\lambda / l), \\
R_{(t)(p)(t)(i)} \equiv R_{M N P Q} t^{M} p^{N} t^{P} n_{i}^{Q}=\mathcal{A}_{i} \cos (\lambda / l), \\
R_{(t)(i)(t)(j)} \equiv R_{M N P Q} t^{M} n_{i}^{N} t^{P} n_{j}^{Q}=\frac{\delta_{i j}}{l^{2}}-\mathcal{M}_{i j} .
\end{gathered}
$$

For the waves in AdS under consideration here, these expressions are exact.

The question is, how do the specific choices of the function $F$ affect the existence, or otherwise, of pp-singularities? It is easy to see that the partially localized solutions with $F=F\left(x^{+}, r\right)$ or $F=F\left(x^{+}, x^{i}\right)$ are indeed singularf as $r \rightarrow 0$. However, one might suspect that there exist fully localized solutions with $F=F\left(x^{+}, x^{i}, r\right)$ that are entirely non-singular. We will see that this is not true.

Absence of pp-singularities requires that all curvature components remain finite as $r \rightarrow 0$. From equation (2.48) and the expression for $\mathcal{A}_{i}$, this implies that $\partial_{r} F=g_{0}\left(r, x^{+}\right)+\mathcal{O}\left(r^{3 \tilde{d} / 2-4}\right)$ as $r \rightarrow 0$, where we are using the notation $f=\mathcal{O}(g)$ to mean that there exists some function $h\left(x^{+}, x^{i}\right)$ such that $f / g \leq h$ as $r \rightarrow 0$. Integrating gives $F=g_{1}\left(r, x^{+}\right)+g_{2}\left(x^{i}, x^{+}\right)+\mathcal{O}\left(r^{3 \tilde{d} / 2-3}\right)$, for some $g_{1}$ and $g_{2}$. Substituting this into the equation of motion for $F$ gives

$$
\frac{1}{r} \frac{\partial}{\partial r}\left(r^{\tilde{d}+1} \frac{\partial g_{1}}{\partial r}\right)+k \nabla_{\perp}^{2} g_{2}=\mathcal{O}\left(r^{5 \tilde{d} / 2-5}\right)
$$

from which it follows that

$$
\nabla_{\perp}^{2} g_{2}=\frac{2}{k}(\tilde{d}-2) F_{1}\left(x^{+}\right)
$$

for some function $F_{1}$. Integrating with respect to $r$ gives

$$
F\left(x^{+}, x^{i}, r\right)=F_{0}\left(x^{+}\right)+\frac{F_{1}\left(x^{+}\right)}{r^{\tilde{d}-2}}+\frac{F_{2}\left(x^{+}\right)}{r^{\tilde{d}}}+g_{2}\left(x^{+}, x^{i}\right)+\mathcal{O}\left(r^{3 \tilde{d} / 2-3}\right) .
$$

\footnotetext{
${ }^{1}$ The reader may be worried that these expressions appear to depend on $\tilde{d}$, which is related to the dimension of the sphere. However, equation (2.2) can be used to eliminate $\tilde{d}$.

${ }^{2}$ The special case $F\left(x^{+}, x^{i}\right)=a_{i}\left(x^{+}\right) x^{i}+F_{0}\left(x^{+}\right)$will be discussed below. Note that the case $F=F\left(x^{+}, x^{i}\right)$ includes the solution $F=H_{i j}\left(x^{+}\right) x^{i} x^{j}$, describing the Randall-Sundrum 21] zero mode, which was previously thought to be non-singular [22]. In this case, we have $\mathcal{M}_{i j} \sim H_{i j}\left(x^{+}\right) / r^{2 \tilde{d}-4}$. Of course, in $\mathrm{AdS}_{3}$ this term vanishes since, in this case, $H_{i j} \rightarrow H_{11}=0$ by virtue of the tracelessness of $H_{i j}$. However, we do not really have a pp-wave in this case, since there can be no propagating gravitational degrees of freedom in three dimensions. In higher dimensions, the zero mode does, in fact, generate singularities at the AdS horizon.
} 
Substituting this into equation (2.47) then yields $F_{2} \equiv 0$ in order for the curvature to remain finite as $r \rightarrow 0$. Finally, substituting into equation (2.49) and requiring finiteness as $r \rightarrow 0$ gives

$$
\frac{\partial^{2} g_{2}}{\partial x^{i} \partial x^{j}}=\frac{\tilde{d}(\tilde{d}-2)}{k d} F_{1}\left(x_{+}\right) \delta_{i j}
$$

which is consistent with equation (2.51). Solving this equation then gives the final result for $F$ :

$$
F\left(x^{+}, x^{i}, r\right)=F_{0}\left(x^{+}\right)+F_{1}\left(x^{+}\right)\left[|x|^{2}+k\left(\frac{d-2}{\tilde{d}-2}\right) \frac{1}{r^{\tilde{d}-2}}\right]+a_{i}\left(x^{+}\right) x^{i}+\Delta\left(x^{+}, x^{i}, r\right),
$$

where we have rescaled $F_{1} . \Delta$ denotes a possible correction term of order $r^{3 \tilde{d} / 2-3}$. Note that $F_{0}$ can be gauged away by redefining $x^{-}$. The $a_{i}\left(x^{+}\right) x^{i}$ term can be also be gauged away, as we shall explain below. So, for the time being, we shall set $F_{0}=a_{i}=0$. We should emphasize that equation (2.54) is the only solution free of pp-singularities. If $\Delta$ is identically zero then this is just the near-horizon solution (2.11) discussed in [7, 25] (with an additional term linear in $x^{i}$ ), i.e., it is an exact solution of the equation of motion (2.10) for pp-waves in AdS. It follows that the correction $\Delta$ must also be an exact solution of equation (2.10), but this correction is negligible near the horizon. If one had just this correction term, then one would also have a solution with no pp-singularities at the horizon. This is easily understood: a solution of $\mathcal{O}\left(r^{3 \tilde{d} / 2-3}\right)$ vanishes at the horizon sufficiently fast that the metric is just like pure AdS as far as geodesics and curvature tensors are concerned[3. In other words, such a solution would represent some perturbation of pure AdS that does not extend as far as the AdS horizon. It seems unlikely that such a solution would correspond to the near-horizon geometry of an intersection of a brane and a pp-wave. The only candidate for such a solution is therefore the solution (2.11). However, it is easy to use the curvature tensors in the appendix to see that this solution is Weyl flat, which implies that it is locally isometric to pure AdS and therefore does not describe a pp-wave in AdS. We shall present the coordinate transformation that gauges away this solution later. Given that this solution is pure gauge, it seems rather doubtful that it describes the near-horizon geometry of a fully localized intersection of a brane and pp-wave, as suggested in [7].

Now, as promised, let us re-instate the term $a_{i}\left(x^{+}\right) x^{i}$. The coordinate transformations that removes the first and second terms in 2.54 do not affect the form of the third term $\left(a_{i}\right.$ is just multiplied by a function of $x^{+}$), and the change in $F$ is the only change in the metric. So once we have removed the first two terms in $F$, we can do the following coordinate transformation:

$$
x^{-} \rightarrow x^{-}+b\left(x^{+}\right)+c_{i}\left(x^{+}\right) x^{i}, \quad x^{i} \rightarrow x^{i}+d^{i}\left(x^{+}\right) .
$$

It is easy to see that $b\left(x^{+}\right), c_{i}\left(x^{+}\right)$and $d_{i}\left(x^{+}\right)$can be chosen to cancel the $a_{i}\left(x^{+}\right) x^{i}$ term in $F$.

To summarize, we have shown that there are no non-singular pp-waves in $\mathrm{AdS}_{d+1}$ with metric of the form (2.14), except possibly solutions that vanish sufficiently fast near the AdS horizon.

\subsection{Brane-waves}

We will now generalize the above analysis to the more complicated case of our brane-waves, described by the metric (2.1). Concentrating on the same curvature components as in (2.47), (2.48) and (2.49) above, in the near-horizon limit, $r \rightarrow 0$, we find

$$
R_{(t)(p)(t)(p)} \rightarrow \frac{1}{l^{2}}-\mathcal{A}_{+} \cos ^{2}(\lambda / l)+\frac{\tilde{d}}{d}(1+\tilde{d}) \frac{r^{\tilde{d}}}{k}\left(\frac{\dot{r}}{r}\right)^{2} \cos ^{2}(\lambda / l),
$$

${ }^{3}$ This is not strictly true in general since second derivatives of $\Delta$ would naively be expected to behave as $r^{3 \tilde{d} / 2-5}$, which vanishes at $r=0$ only if $\tilde{d}>3$, i.e., $d<6$. However, it is probably possible to derive stronger bounds on $\Delta$. 


$$
\begin{gathered}
R_{(t)(p)(t)(i)} \rightarrow \mathcal{A}_{i} \cos (\lambda / l), \\
R_{(t)(i)(t)(j)} \rightarrow \frac{\delta_{i j}}{l^{2}}-\mathcal{M}_{i j}+\delta_{i j} \frac{\tilde{d}}{d}(1+\tilde{d}) \frac{r^{\tilde{d}}}{k}\left(\frac{\dot{r}}{r}\right)^{2} .
\end{gathered}
$$

Note that there are now extra terms involving $\dot{r}$. It possible that these terms could cancel the divergences coming from the other terms. This emphasizes the importance of examining the full brane-wave spacetime, and not just the near-horizon geometry. We have retained only the leading order divergences, and assumed that $\dot{r}$ diverges as $r \rightarrow 0$. (If $\dot{r}$ remains finite then the extra contributions above are clearly finite and cancellations will not occur.)

Using equation (2.57), we can obtain equation (2.52) as above. However, there will now be an extra $\mathcal{O}\left(r^{2}\right)$ correction term on the right hand side, arising from neglecting the 1 in $H$ in deriving equation (2.10). This is subleading order for the M5-brane but leading order for the other branes ${ }^{4}$.

It is now possible that the term in $F$ of order $r^{-\tilde{d}}$ may be consistent with finite curvature provided that the $\dot{r}$ terms cancel the divergences arising from this term. Equation (2.56) shows that $\dot{r}$ would have to be of order $r^{2-3 \tilde{d} / 2}$ as $r \rightarrow 0$ in order to cancel the divergence in $\mathcal{A}_{+}$. To see whether this is possible, we need to examine the geodesic equation (2.34). The dominant term on the right hand side would be the second term (involving $\dot{r}^{2}$ ). Neglecting the other terms, and integrating then gives $\dot{r} \sim r^{2-\tilde{d} / 2}$ (this could also be obtained from equation (2.35)), which is a contradiction. It is therefore not possible for the $\dot{r}$ terms to cancel the divergence arising from the $r^{-\tilde{d}}$ term in $F$, so $F_{2}$ has to be set to zero in order to obtain finite curvature, just as above.

Now consider equation (2.58). An identical argument shows that the $\dot{r}$ term cannot cancel the divergence coming from $\mathcal{M}_{i j}$, so one obtains equation (2.53) as above. Putting everything together, one obtains the same solution (2.54) but with the exception that the correction term is $\mathcal{O}\left(r^{2}\right)$ for the M2 and D3 branes. (Note that such a term occurs in equation (2.12).)

Thus, we conclude that the only non-singular brane-waves must have $F$ of the form (2.11) near the brane. Since this form for $F$ can be gauged away, we conclude that there are no non-singular pp-waves on the non-dilatonic branes, of the supergravity theories that we have been considering. This

is reminiscent of the results concerning waves on strings in five and six dimensions [2, 3, 代, in which similar singularities in the curvature components at the horizon of the string were found.

\section{Virasoro symmetry}

Before moving on to the cases of interest to us, we will review some relevant points concerning $\mathrm{AdS}_{3}$ and its two-dimensional CFT dual.

\section{1 $\mathbf{A d S}_{3}$, Virasoro symmetry and $(2+1)$-dimensional "pp-waves"}

Consider $\mathrm{AdS}_{3}$ in horospherical coordinates

$$
d s^{2}=\frac{l^{2}}{z^{2}}\left(d x^{+} d x^{-}+d z^{2}\right) .
$$

Since $\mathrm{AdS}_{3}$ is the group manifold $S L(2, \mathbb{R})$, the metric (3.1) has the isometry group $S L(2, \mathbb{R})_{L} \otimes$ $S L(2, \mathbb{R})_{R}=S O(2,2)$. The $S L(2, \mathbb{R})_{L}$ action is given by:

$$
x^{+} \rightarrow f\left(x^{+}\right)
$$

\footnotetext{
${ }^{4}$ Once again, it may be possible to derive better bounds on the correction term.

${ }^{5}$ We have rescaled $x^{ \pm}$by a factor of $\sqrt{2}$.
} 


$$
\begin{aligned}
x^{-} & \rightarrow x^{-}-\frac{1}{2} z^{2} \frac{f^{\prime \prime}}{f^{\prime}}, \\
z & \rightarrow z \sqrt{f^{\prime}}
\end{aligned}
$$

where

$$
f\left(x^{+}\right)=\frac{a x^{+}+b}{c x^{+}+d} \in S L(2, \mathbb{R})
$$

and a prime denotes a derivative with respect to $x^{+}$. Note that elements of $S L(2, \mathbb{R})$ have a vanishing Schwarzian derivative:

$$
\left\{f, x^{+}\right\}=\frac{f^{\prime \prime \prime}}{f^{\prime}}-\frac{3}{2}\left(\frac{f^{\prime \prime}}{f^{\prime}}\right)^{2}=0 .
$$

The $S L(2, \mathbb{R})_{R}$ action has the same form as (3.2), but with $x^{+}$and $x^{-}$interchanged:

$$
\begin{aligned}
x^{-} & \rightarrow f\left(x^{-}\right), \\
x^{+} & \rightarrow x^{+}-\frac{1}{2} z^{2} \frac{f^{\prime \prime}}{f^{\prime}}, \\
z & \rightarrow z \sqrt{f^{\prime}},
\end{aligned}
$$

where now

$$
f\left(x^{-}\right)=\frac{a x^{-}+b}{c x^{-}+d} \in S L(2, \mathbb{R}),
$$

and a prime denotes a derivative with respect to $x^{-}$. This isometry group acts on the boundary as the global conformal group of two-dimensional Minkowski space, generated by two copies of the Virasoro generators $L_{0}$ and $L_{ \pm 1}$.

Now the AdS/CFT correspondence asserts that the AdS isometry group is identical to the conformal group on the boundary. In two dimensions, the latter is (two copies of) the infinite dimensional Virasoro group, so there is a puzzle here: how can the finite-dimensional isometry group be equivalent to the infinite-dimensional conformal group on the boundary? The puzzle was resolved long ago by Brown and Henneaux [14]. The isometries (3.2) and (3.6) leave the metric unchanged, and the corresponding generators will annihilate the vacuum state in the quantum theory. These isometries must, however, be supplemented by the infinite number of diffeomorphisms which change the metric, but leave the asymptotic form of $\mathrm{AdS}_{3}$ invariant up to a conformal factor. An analysis of the (modes of the) generators of these allowed diffeomorphisms shows that they obey (two copies of) the Virasoro algebra. The asymptotic symmetry group of $\mathrm{AdS}_{3}$ is thus just the conformal group in $1+1$ dimensions, as expected.

The induced metric on the boundary is

$$
d s^{2}=\frac{l^{2}}{z^{2}} d x^{+} d x^{-}
$$

the conformal transformations of which are $x^{+} \rightarrow f\left(x^{+}\right)$and $x^{-} \rightarrow f\left(x^{-}\right)$. In the bulk, then, the asymptotic structure-preserving diffeomorphisms are precisely those of (3.2) and (3.6) but for arbitrary functions $f\left(x^{+}\right)$and $f\left(x^{-}\right)$. By arbitrary here, we mean $\left\{f, x^{+}\right\},\left\{f, x^{-}\right\} \neq 0$. Under the former transformation, we find

$$
d s^{2} \rightarrow \frac{l^{2}}{z^{2}}\left(d x^{+} d x^{-}+F\left(x^{+}\right) z^{2} d x^{+2}+d z^{2}\right)
$$

where

$$
F\left(x^{+}\right)=-\frac{1}{2}\left\{f, x^{+}\right\}
$$


As promised, these general transformations are not isometries of the metric, but do leave the boundary manifold unchanged up to a conformal factor (equal to $f^{\prime}$ ).

There are two points to note about the metric (3.8). Firstly, it looks like a pp-wave in $\mathrm{AdS}_{3}$. However, there are no propagating degrees of freedom in $(2+1)$-dimensional gravity, so what we mean by this is the following. The metric of a wave in $\mathrm{AdS}_{3}$ would be of the form

$$
d s^{2}=\frac{l^{2}}{z^{2}}\left(d x^{+} d x^{-}+F\left(x^{+}, z\right) d x^{+2}+d z^{2}\right),
$$

which is a solution of Einstein's equations with negative cosmological constant if and only if

$$
\partial_{z}\left(\frac{1}{z} \partial_{z} F\right)=0
$$

implying

$$
F\left(x^{+}, z\right)=F_{0}\left(x^{+}\right) z^{2}+F_{1}\left(x^{+}\right),
$$

for arbitrary functions $F_{0}$ and $F_{1}$. Now, $F_{1}$ can be gauged away by a redefinition of $x^{-}$, leaving a metric of the form (3.8), which we know is obtained from pure $\mathrm{AdS}_{3}$ by a coordinate transformation of the form (3.2) with arbitrary $f\left(x^{+}\right)$. Therefore there are no pp-waves in $\mathrm{AdS}_{3}$, as expected.

The metric (3.8) is locally isometric to $\mathrm{AdS}_{3}$. This is reminiscent of the BTZ black hole [23, 24]. In fact, when $F\left(x^{+}\right)=$constant, it is straightforward to obtain the extreme BTZ black hole from the metric (3.8) 25]. To see this, we compactify along the direction of propagation of the wave, taking the period to be $2 \pi$. Calling this coordinate $\phi$, taking $F\left(x^{+}\right)=8 G M l^{2}$, and rewriting in terms of $r=1 / z$, the metric (3.8) becomes

$$
\frac{d s^{2}}{l^{2}}=-\left(r^{2}-4 G M l^{2}\right) d t^{2}+\left(r^{2}+4 G M l^{2}\right) d \phi^{2}-4 G M l^{2} d t d \phi+\frac{d r^{2}}{r^{2}}
$$

Rescaling $t \rightarrow l^{2} t$ and $\phi \rightarrow l \phi$ gives the metric of the extreme $M l=J$ BTZ black hole [23, 24]:

$$
d s^{2}=-N^{2} d t^{2}+\rho^{2}\left(N^{\phi} d t+d \phi\right)^{2}+\frac{r^{2}}{N^{2} \rho^{2}} d r^{2}
$$

where

$$
\begin{gathered}
\rho^{2}=r^{2}+4 G M l^{2}, \\
N^{2}=r^{4} /(l \rho)^{2}, \\
N^{\phi}=-4 G M l / \rho^{2} .
\end{gathered}
$$

So there is a nice correspondence: by periodically identifying one of the coordinates, $\mathrm{AdS}_{3}$ in Poincaré coordinates becomes the $M=J=0$ BTZ black hole [23, 24]; and a "wave" in $\mathrm{AdS}_{3}$ becomes the extreme $M l=J$ BTZ hole [9, 25].

\subsection{Virasoro symmetry in higher dimensions}

The isometry group of $\mathrm{AdS}_{3}$ combines with a subgroup of general diffeomorphisms to give the Virasoro group. In higher dimensions this is not possible. However, it has recently been shown [15] that the space of metrics of the form

$$
d s^{2}=\frac{l^{2}}{z^{2}}\left(d x^{+} d x^{-}+F\left(x^{+}, x^{i}, z\right) d x^{+^{2}}+d x^{i} d x^{i}+d z^{2}\right)
$$


does possess an infinite dimensional symmetry group, namely

$$
\begin{aligned}
x^{+} & \rightarrow f\left(x^{+}\right), \\
x^{-} & \rightarrow x^{-}-\frac{1}{2}\left(|x|^{2}+z^{2}\right) \frac{f^{\prime \prime}}{f^{\prime}}, \\
x^{i} & \rightarrow x^{i} \sqrt{f^{\prime}}, \\
z & \rightarrow z \sqrt{f^{\prime}},
\end{aligned}
$$

with the function $F$ shifting according to

$$
F\left(x^{+}, x^{i}, z\right) \rightarrow F\left(x^{+}, x^{i}, z\right) f^{\prime}-\frac{1}{2}\left(|x|^{2}+z^{2}\right)\left\{f, x^{+}\right\},
$$

with the Schwarzian derivative defined in (3.4). If one starts from pure $\operatorname{AdS}_{d+1}(F \equiv 0)$ then the change of coordinates (3.16) takes one to a pp-wave metric with

$$
F\left(x^{+}, x^{i}, z\right)=F\left(x^{+}\right)\left(|x|^{2}+z^{2}\right),
$$

for some arbitrary $F\left(x^{+}\right)$. Writing this in terms of the coordinate $r$ and using the relation (2.2) brings $F$ to the form (2.11) without the $F_{0}$ and $a_{i} x^{i}$ pieces (which can be gauged away anyway). Therefore the solution (2.11) just describes pure AdS in unusual coordinates, which justifies our claim that this solution is pure gauge.

It was proved in 15 that the transformations (3.16) do indeed generate the Virasoro group. It is natural to ask why this symmetry exists. To answer this question, we shall need to discuss two different sets of coordinate on AdS. First, recall that AdS is defined as a hyperboloid embedded in flat space with two time directions:

$$
\begin{gathered}
d s^{2}=d u d v+d X^{+} d X^{-}+d X^{i} d X^{i}, \\
u v+X^{+} X^{-}+X^{i} X^{i}=-l^{2} .
\end{gathered}
$$

If one now defines $x^{ \pm}=X^{ \pm} / u$ and $x^{i}=X^{i} / u$, and eliminates $v$, then one obtains the metric for AdS in horospherical coordinates:

$$
d s^{2}=l^{2} \frac{d u^{2}}{u^{2}}+u^{2}\left(d x^{+} d x^{-}+d x^{i} d x^{i}\right)
$$

A second set of coordinate on AdS can be constructed based on the observation that the surfaces $X^{i}=$ constant have $\mathrm{AdS}_{3}$ geometry. Define new coordinates by $X^{1}=l \sinh y \cos \theta_{1}, X^{2}=l \sinh y \cos \theta_{1} \sin \theta_{2}$ etc., $u=l \cosh y \tilde{u}, v=l \cosh y \tilde{v}, X^{ \pm}=l \cosh y \tilde{X}^{ \pm}$, which gives

$$
\begin{gathered}
d s^{2}=l^{2}\left(d y^{2}+\cosh ^{2} y\left(d \tilde{u} d \tilde{v}+d \tilde{X}^{+} d \tilde{X}^{-}\right)+\sinh ^{2} y d \Omega^{2}\right), \\
\tilde{u} \tilde{v}+\tilde{X}^{+} \tilde{X}^{-}=-1,
\end{gathered}
$$

which makes it clear that surfaces of constant $y$ and $\Omega$ are $\mathrm{AdS}_{3}$ hyperboloids. Now introduce horospherical coordinate on each of these hyperboloids: $x^{ \pm}=\tilde{X}^{ \pm} / \tilde{u}$ and eliminate $\tilde{v}$ to get

$$
d s^{2}=l^{2}\left(d y^{2}+\cosh ^{2} y\left(\frac{d \tilde{u}^{2}}{\tilde{u}^{2}}+\tilde{u}^{2} d x^{+} d x^{-}\right)+\sinh ^{2} y d \Omega^{2}\right) .
$$

It is straightforward to work out how the coordinate systems used in (3.21) and (3.24) are related. The coordinates $x^{ \pm}$are the same for the two metrics. The other coordinates are related by $u=l \cosh y \tilde{u}$ and $x^{1} u=l \sinh y \cos \theta_{1}$ etc. 
Consider now a pp-wave spacetime, with metric

$$
\begin{aligned}
d s^{2} & =l^{2} \frac{d u^{2}}{u^{2}}+u^{2}\left(d x^{+} d x^{-}+F\left(x^{+}, x^{i}, u\right) d x^{+^{2}}+d x^{i} d x^{i}\right) \\
& =d s^{2}(A d S)+u^{2} F\left(x^{+}, x^{i}, u\right) d x^{+^{2}} .
\end{aligned}
$$

This is written in the horospherical coordinates. Now transform to the other coordinate system:

$$
\begin{aligned}
d s^{2} & =d s^{2}(A d S)+l^{2} \cosh ^{2} y \tilde{u}^{2} F d x^{+^{2}} \\
& =l^{2}\left(d y^{2}+\cosh ^{2} y\left(\frac{d \tilde{u}^{2}}{\tilde{u}^{2}}+\tilde{u}^{2}\left(d x^{+} d x^{-}+F d x^{+^{2}}\right)\right)+\sinh ^{2} y d \Omega^{2}\right) .
\end{aligned}
$$

It now looks like the surfaces of constant $y$ and $\Omega$ have the geometry of pp-waves in $\mathrm{AdS}_{3}$, which were discussed above. However, we have to be slightly careful because $F$ depends on the coordinates $y$ and $\Omega$, so these pp-waves will be off-shell in three dimensions (i.e., $F$ will not satisfy equation (3.11)). Now consider the Virasoro symmetry (3.16) acting on the metric (3.25) (with $u=l / z$ ). Writing this in terms of the new coordinates gives

$$
\begin{aligned}
x^{+} & \rightarrow f\left(x^{+}\right), \\
x^{-} & \rightarrow x^{-}-\frac{1}{2 \tilde{u}^{2}} \frac{f^{\prime \prime}}{f^{\prime}}, \\
\tilde{u} & \rightarrow \frac{\tilde{u}}{\sqrt{f^{\prime}}},
\end{aligned}
$$

with $y$ and $\Omega$ unchanged and

$$
F \rightarrow F f^{\prime}-\frac{1}{2 \tilde{u}^{2}}\left\{f, x^{+}\right\}
$$

This is clearly nothing but the Virasoro symmetry acting on the three dimensional surfaces of constant $y$ and $\Omega$. Thus the existence of the higher dimensional Virasoro symmetry arises from the existence of the Virasoro symmetry for three dimensional asymptotically AdS submanifolds 0 .

Note that the higher dimensional pp-wave (3.15) can only be asymptotic to AdS when $F \rightarrow 0$ as $z \rightarrow 0$. More general pp-waves will not have Minkowski space as their conformal boundary at infinity, and therefore be of less interest from the point of view of AdS/CFT. A Virasoro transformation will introduce a term $|x|^{2}\left\{f, x^{+}\right\}$into $F$. This destroys the preferred coordinate system on the boundary at infinity (although the boundary is, of course, still flat space since this is only a coordinate transformation), and therefore does not correspond to a conformal transformation on the boundary. The only transformations that preserve the preferred flat coordinates on the boundary are those with $\left\{f, x^{+}\right\}=0$, which give an $S L(2, R)$ subgroup of the conformal group on the boundary.

\section{Boundary energy-momentum tensor}

We have shown that a pp-wave propagating on a non-dilatonic brane has pp-curvature singularities at the horizon. The near-horizon geometry of such spacetimes is a pp-wave in AdS space and we will now consider the AdS/CFT correspondence in such cases. If this makes sense then the CFT provides a resolution of the singularity. The easiest thing to compute is the CFT energy-momentum tensor,

\footnotetext{
${ }^{6}$ Actually these submanifold are only asymptotically AdS when $F \rightarrow 0$ as $\tilde{u} \rightarrow \infty$, which implies $F \rightarrow 0$ as $u \rightarrow \infty$, which implies that the higher dimensional spacetime is also asymptotically AdS. If this requirement is not satisfied then the action of the Virasoro symmetry on the three dimensional submanifolds is a generalization of the usual action to certain spacetimes that are not asymptotic to $\mathrm{AdS}_{3}$.
} 
making use of the boundary counterterm method of [13, 27, 28, 29, 30, 31, 32]. Our starting point is the metric (3.15), describing a pp-wave in $\mathrm{AdS}_{d+1}$. The boundary is at $z=0$, and the induced metric on surfaces of constant $z$ is

$$
\left.d s^{2}\right|_{z=\text { const. }}=g_{i j}(x) d x^{i} d x^{j}=\frac{l^{2}}{z^{2}}\left(d x^{+} d x^{-}+F\left(x^{+}, x^{i}, z\right) d x^{+^{2}}+d x^{i} d x^{i}\right),
$$

where $i, j=0, \ldots, d-1$. The outward unit normal to such a surface is $n=-(l / z) d z$. Using the formulae from [32], the energy-momentum tensor is given by

$$
<T_{i j}>=\frac{1}{8 \pi G_{d+1}}\left[-\left(K_{i j}-K g_{i j}\right)-\frac{d-1}{l} g_{i j}+\frac{l}{d-2}\left(R_{i j}-\frac{1}{2} R g_{i j}\right)+\ldots\right],
$$

where $R_{i j}$ and $R$ are the Ricci tensor and Ricci scalar associated with $g_{i j}$ and $K_{i j}$ denotes the tangential components of the extrinsic curvature of the boundary, defined by

$$
K_{\mu \nu}=h_{\mu}^{\rho} h_{\nu}^{\sigma} \nabla_{\rho} n_{\sigma}
$$

where $h_{\nu}^{\mu}=\delta_{\nu}^{\mu}-n^{\mu} n_{\nu}$. For $d \geq 6$ one needs further counterterms.

This calculation will yield the CFT energy-momentum tensor in the conformal frame where the metric is $g_{i j}$, which includes the prefactor $l^{2} / z^{2}$. To get rid of this factor, one must perform a Weyl transformation to obtain the metric $\bar{g}_{i j}=\left(z^{2} / l^{2}\right) g_{i j}$. In the new conformal frame, the energy momentum tensor is $\bar{T}_{i j}=(l / z)^{d-2} T_{i j}$ [33], and the metric is

$$
d s^{2}=\bar{g}_{i j} d x^{i} d x^{j}=d x^{+} d x^{-}+F\left(x^{+}, x^{i}, 0\right) d x^{+^{2}}+d x^{i} d x^{i},
$$

which is manifestly flat if $F\left(x^{+}, x^{i}, 0\right)=0$, which is what we shall usually assume. The counterterm calculation is straightforward. The only non-vanishing component of the CFT energy-momentum tensor turns out to be

$$
<\bar{T}_{++}>=\frac{l^{d-1}}{16 \pi G_{d+1}} \lim _{z \rightarrow 0} \frac{1}{z^{d-1}}\left[\frac{\partial F}{\partial z}-\frac{z}{d-2} \nabla_{\perp}^{2} F-\frac{z^{3}}{(d-2)^{2}(d-4)}\left(\nabla_{\perp}^{2}\right)^{2} F+\ldots\right],
$$

where we have included the first three boundary counterterms. However, not all three are present for low dimensional examples. For $d=2$, only the first term above should be kept; for $d=3$, 4 , the first two terms are needed, one keeps the first two terms; and for $d=5,6$, one needs the third term too. Higher order terms would occur in higher dimensional examples.

Finally, we must write everything in terms of the parameters of the dual CFT. For $d=2$, the CFT has central charge $c=3 l /\left(2 G_{3}\right)$ [14, so we obtain

$$
<T_{++}>=\frac{c}{24 \pi} \lim _{z \rightarrow 0} \frac{1}{z} \frac{\partial F}{\partial z}
$$

where we have dropped the bar on $T_{++}$since we shall always be working in the same conformal frame. In the examples obtained from non-dilatonic branes, namely $d=3,4,6$, the following formulae apply [8]

$$
\begin{gathered}
\frac{l^{2}}{G_{4}}=\frac{2 \sqrt{2} N^{3 / 2}}{3}, \\
\frac{l^{3}}{G_{5}}=\frac{2 N^{2}}{\pi}, \\
\frac{l^{5}}{G_{7}}=\frac{16 N^{3}}{3 \pi^{2}},
\end{gathered}
$$


where $N$ is the number of branes present before the decoupling limit is taken. Substituting into $<T_{++}>$then gives

$$
\begin{gathered}
<T_{++}>=\frac{\sqrt{2} N^{3 / 2}}{24 \pi} \lim _{z \rightarrow 0} \frac{1}{z^{2}}\left[\frac{\partial F}{\partial z}-z \nabla_{\perp}^{2} F\right], \\
<T_{++}>=\frac{N^{2}}{8 \pi^{2}} \lim _{z \rightarrow 0} \frac{1}{z^{3}}\left[\frac{\partial F}{\partial z}-\frac{z}{2} \nabla_{\perp}^{2} F\right], \\
<T_{++}>=\frac{N^{3}}{3 \pi^{3}} \lim _{z \rightarrow 0} \frac{1}{z^{5}}\left[\frac{\partial F}{\partial z}-\frac{z}{4} \nabla_{\perp}^{2} F-\frac{z^{3}}{32}\left(\nabla_{\perp}^{2}\right)^{2} F\right]
\end{gathered}
$$

for $d=3,4,6$ respectively.

The energy-momentum tensor is obviously dependent on the form of the function $F$. In particular, for the spurious wave with $F$ as in (3.18), we have $\left\langle T_{++}\right\rangle=0$, which is in agreement with the fact that our spacetime in this case is just pure AdS in unusual coordinates. Secondly, a Ricci-flat brane, with $F=F\left(x^{+}, x^{i}\right)$, also has $\left\langle T_{++}>=0\right.$ since the Einstein equations in this case give $\nabla_{\perp}^{2} F=0$.

In general, the function $F$ will have $z$-dependence that is a linear combination of $z^{\frac{d}{d}}$ and $z^{0}$ as $z \rightarrow 0$. This can be seen by Fourier analyzing equation (2.9) with respect to $x^{i}$ to obtain an equation in $z$ that can be solved with Bessel functions [22]. Solutions proportional to $z^{d}$ give finite $\left\langle T_{++}\right\rangle$ whereas those proportional to $z^{0}$ give a divergent result in general. This is just the distinction between normalizable and non-normalizable bulk modes [34], with the former corresponding to a deformation of the state of the CFT and the latter to a change in the parameters of the CFT Lagrangian, in this case the boundary metric. We shall be interested only in the bulk solutions proportional to $z^{d}$ at the boundary, for which the boundary is flat space and the CFT is in a quantum state with non-vanishing $<T_{++}>$, i.e., a null momentum density. In fact, we shall restrict ourselves to solutions of the form

$$
F\left(x^{+}, x^{i}, z\right)=F\left(x^{+}\right) z^{d} .
$$

The boundary energy-momentum tensor then describes a disturbance propagating at the speed of light in the negative $x$-direction with wave profile determined by $F\left(x^{+}\right)$.

The case $F\left(x^{+}\right)=$constant was studied in [9]. Starting from a non-extremal stack of branes, it was shown that a finite boost produces a metric describing a pp-wave propagating on the branes. However, in order to retain the pp-wave in the extremal limit, it is necessary to take the boost parameter to infinity. Therefore, the metric (2.1) with $F\left(x^{+}, x^{i}, z\right)=Q / r^{\tilde{d}}(Q=$ constant) can be regarded as describing an infinitely boosted stack of BPS branes. Taking the decoupling limit keeping the momentum finite, one obtains a sphere times an asymptotically AdS pp-wave spacetime with

$$
F\left(x^{+}, x^{i}, z\right)=\mu^{d} z^{d},
$$

where $\mu$ is a constant. In four dimensions, this pp-wave spacetime was first discussed by Kaigorodov [10, 11]. Its $D$-dimensional generalization, denoted $K_{D}$, was presented in [9]. The above discussion shows that $K_{D}$ can be regarded as an infinitely boosted version of $\operatorname{AdS}_{D}$. The infinite boost in the bulk induces an infinite boost on the conformal boundary, and it is therefore natural to conjecture that supergravity theory in the Kaigorodov spacetime (times a sphere of appropriate dimension) is dual to a strongly coupled large $N$ CFT in an infinitely boosted frame with constant background momentum density [9].

The Kaigorodov spacetime is a special case of the class of pp-wave spacetimes that we have been considering, and we have seen that all of these give a CFT energy-momentum tensor that describes a null momentum density in the $x$-direction. In the case of $K_{d+1}$, the momentum density is in fact constant: $P=<T_{++}>$is given by

$$
P=\frac{c \mu^{2}}{12 \pi}
$$




$$
\begin{gathered}
P=\frac{\sqrt{2} N^{3 / 2} \mu^{3}}{8 \pi}, \\
P=\frac{N^{2} \mu^{4}}{2 \pi^{2}}, \\
P=\frac{2 N^{3} \mu^{6}}{\pi^{3}},
\end{gathered}
$$

for $d=2,3,4,6$ respectively. Note that a null momentum density is precisely what one would expect from an infinite boost. Our results are therefore evidence in favour of the conjecture of [9]. The classical supergravity approximation is valid in the large $N$ limit, in which case $P \rightarrow \infty$.

The three-dimensional Kaigorodov spacetime, $K_{3}$, is just that described by the metric (3.10), with $F\left(x^{+}, z\right)=\mu^{2} z^{2}$, and it is curious that the energy-momentum tensor in this case is non-vanishing, since we know from the above discussion that this spacetime is just pure $\mathrm{AdS}_{3}$ in unusual coordinates. The result can be understood as follows [35]. Start with pure $\mathrm{AdS}_{3}$ in horospherical coordinates, given by (3.1). This corresponds to the $S L(2, \mathbb{R})_{L} \otimes S L(2, \mathbb{R})_{R}$ vacuum of the CFT, and the corresponding energy-momentum tensor will therefore vanish. As we have seen, $K_{3}$ can be obtained from this metric via the coordinate transformation (3.2), with

$$
F\left(x^{+}\right)=-\frac{1}{2}\left\{f, x^{+}\right\}=\mu^{2},
$$

a solution of which is

$$
f\left(x^{+}\right)=\frac{1}{2 \mu} e^{2 \mu x^{+}} .
$$

The new boundary coordinates are $x^{+^{\prime}}=f\left(x^{+}\right)$and $x^{{ }^{\prime}}=x^{-}$. The boundary metric is

$$
d s^{2}=d x^{+} d x^{-}=e^{2 \mu x^{+^{\prime}}} d x^{+^{\prime}} d x^{-^{\prime}} .
$$

The AdS/CFT calculation in $K_{3}$ gives the energy-momentum tensor in the conformal frame where the metric is

$$
d s^{2}=d x^{+\prime} d x^{-\prime}
$$

which can be obtained from the metric $(4.21)$ by a Weyl transformation. Under the Weyl transformation, the energy-momentum tensor transforms as [36]:

$$
T_{+^{\prime}+^{\prime}}^{\prime}=T_{+^{\prime}+^{\prime}}-\frac{c}{24 \pi}\left\{f, x^{+^{\prime}}\right\}
$$

Since $T_{+^{\prime}+^{\prime}}$ vanishes, this gives

$$
T_{+^{\prime}+^{\prime}}^{\prime}=\frac{c \mu^{2}}{12 \pi}
$$

which is precisely what we obtained from our AdS/CFT calculation. Note that the Kaigorodov coordinates cover only the region $x^{+}>0$ of the original spacetime. This is very similar to what occurs for the BTZ black hole, and corresponds to adopting the coordinates of an accelerating observer on the boundary. Such an observer would naturally define the energy-momentum tensor so that it vanished in the vacuum defined with respect to his time coordinate. This energy-momentum tensor has a finite expectation value in the $S L(2, R) \times S L(2, R)$ vacuum state and describes the thermal radiation experienced by such an observer [35].

Having understood the $\mathrm{AdS}_{3}$ case, we now move on to discuss the higher-dimensional generalizations of the Kaigorodov spacetime.

\footnotetext{
${ }^{7}$ We have inserted a factor of $-1 /(2 \pi)$ relative to the string theory convention 36 in order to be consistent with the usual Lorentzian field theory definition of the energy-momentum tensor.
} 


\section{The Kaigorodov spacetime}

\subsection{The metric and its symmetries}

The $(d+1)$-dimensional Kaigorodov metric $K_{d+1}$ can be written

$$
d s^{2}=\frac{l^{2}}{z^{2}}\left(d x^{+} d x^{-}+\mu^{d} z^{d} d x^{+^{2}}+d x^{i} d x^{i}+d z^{2}\right),
$$

where $i=1 \ldots d-2$. It will sometimes be useful to write

$$
x^{ \pm}=x \pm t .
$$

The Kaigorodov spacetime is clearly asymptotic to $\mathrm{AdS}_{d+1}$ as $z \rightarrow 0$, and has a pp-curvature singularity as $z \rightarrow \infty$. It was shown in 9] that this metric preserves $1 / 4$ supersymmetry, and has the following Killing vector fields (we have converted the results of 90 to our coordinate system)

$$
\begin{gathered}
K_{+}=\sqrt{2} \frac{\partial}{\partial x^{+}}, \quad K_{-}=\sqrt{2} \frac{\partial}{\partial x^{-}}, \quad K_{i}=\frac{\partial}{\partial x^{i}}, \\
L_{i j}=x^{i} \frac{\partial}{\partial x^{j}}-x^{j} \frac{\partial}{\partial x^{i}}, \\
L_{i}=\frac{x^{+}}{\sqrt{2}} \frac{\partial}{\partial x^{i}}-\sqrt{2} x^{i} \frac{\partial}{\partial x^{-}}, \\
J=-\frac{z}{l} \frac{\partial}{\partial z}-\frac{(d+2) x^{-}}{2 l} \frac{\partial}{\partial x^{-}}+\frac{(d-2) x^{+}}{2 l} \frac{\partial}{\partial x^{+}}-\frac{x^{i}}{l} \frac{\partial}{\partial x^{i}} .
\end{gathered}
$$

The commutation relations of these Killing vector fields were presented in [9].

\subsection{Matching of symmetries}

The above Killing vector fields induce symmetries of the boundary theory. In this subsection, we shall show that these symmetries are the symmetries that one would expect for a CFT in a background with the conformal symmetry broken by a constant (null) momentum density in the $x^{+}$direction. As we saw above, such a momentum density is described by an energy-momentum tensor, the only non-vanishing component of which is $P \equiv<T_{++}>=$constant. The symmetries of the boundary theory are simply those conformal transformations that leave this energy-momentum tensor invariant.

Some of the symmetries are obvious: the $K$ generators just give boundary translations, and the $L_{i j}$ correspond to transverse rotations. These transformations clearly leave the boundary metric invariant, and preserve the form of the background energy-momentum tensor. The other generators are more complicated. Let $y=x^{1}$ and consider $L_{y}$. The finite form of the bulk isometry generated by this Killing vector field is

$$
\begin{aligned}
t^{\prime} & =\left(1+\frac{\lambda^{2}}{4}\right) t+\frac{\lambda^{2}}{4} x+\frac{\lambda}{\sqrt{2}} y, \\
x^{\prime} & =\left(1-\frac{\lambda^{2}}{4}\right) x-\frac{\lambda^{2}}{4} t-\frac{\lambda}{\sqrt{2}} y \\
y^{\prime} & =y+\frac{\lambda}{\sqrt{2}} t+\frac{\lambda}{\sqrt{2}} x .
\end{aligned}
$$

This is a combination of a boost in the $y$-direction, a rotation in the $x y$ plane and a boost in the $x$-direction. Clearly the parameters of the three transformations are related. To see how this emerges, 
consider the transformations separately. A boost with velocity $v$ in the $y$-direction followed by a rotation through angle $\theta$ in the $x y$ plane gives

$$
x^{+^{\prime}}=\frac{1}{2}(\cos \theta+\gamma+\gamma v \sin \theta) x^{+}+\frac{1}{2}(\cos \theta-\gamma-\gamma v \sin \theta) x^{-}-\gamma(\sin \theta+v) y,
$$

where $\gamma=\left(1-v^{2}\right)^{-1 / 2}$. The energy momentum tensor after this transformation becomes

$$
<T_{\mu^{\prime} \nu^{\prime}}>=\frac{\partial x^{+}}{\partial x^{\mu \prime}} \frac{\partial x^{+}}{\partial x^{\nu^{\prime}}}<T_{++}>
$$

so if the direction of momentum flow is to remain unchanged then we require $x^{+}$to be independent of $x^{-^{\prime}}$ and $y^{\prime}$. Inverting equation 5.8 (by replacing $v \rightarrow-v$ and $\theta \rightarrow-\theta$ ), this requirement reduces to

$$
v=-\sin \theta, \quad \cos \theta>0 .
$$

The new energy momentum tensor then has only one non-vanishing component, viz

$$
<T_{+^{\prime}+^{\prime}}>=\cos ^{2} \theta<T_{++}>
$$

Finally, the $\cos ^{2} \theta$ prefactor can be removed by boosting along the $x$-direction with velocity $w$, which gives

$$
x^{+^{\prime \prime}}=\sqrt{\frac{1-w}{1+w}} x^{+^{\prime}}
$$

so

$$
<T_{+{ }^{\prime \prime}+}{ }^{\prime \prime}>=\frac{1+w}{1-w} \cos ^{2} \theta<T_{++}>
$$

hence if $w=\sin ^{2} \theta /\left(1+\cos ^{2} \theta\right)$ then the energy momentum tensor in the new frame is the same as in the original frame. Therefore, the combined transformation is a symmetry of the background of the boundary theory. In simple terms, it can be understood as follows ${ }^{8}$. In coordinates $t, x, x^{i}$, the momentum density is $E(1,1,0, \ldots 0)$. (This is null because we are working in the infinite momentum frame.) The boost in the $y$-direction takes this to $E^{\prime}(1, \cos \theta,-\sin \theta, \ldots 0)$ for some energy $E^{\prime}$. The rotation in the $x y$ plane brings this to $E^{\prime}(1,1,0, \ldots 0)$. Finally, the boost in the $x$-direction brings this back to to $E(1,1,0 \ldots 0)$.

The symmetries that we have discussed so far form the subgroup of the Poincaré group that is unbroken by the background momentum density. The remaining generator $J$ involves an unbroken conformal symmetry. The finite bulk isometry induced by $J$ is

$$
x^{+^{\prime}}=\lambda \lambda^{-d / 2} x^{+}, \quad x^{-^{\prime}}=\lambda \lambda^{d / 2} x^{-}, \quad, x^{i^{\prime}}=\lambda x^{i}, \quad z^{\prime}=\lambda z
$$

which is clearly a combination of a dilatation by factor $\lambda$, and the transformation

$$
x^{ \pm} \rightarrow \lambda^{\mp d / 2} x^{ \pm}
$$

This latter transformation is simply a boost in the $x$-direction. To see why this combination of dilatation and boost is a symmetry of the boundary theory, consider replacing the boost by a general boost in the $x$-direction with velocity $v$. For the boundary theory the combined dilatation and boost is

$$
x^{ \pm \prime}=\lambda \sqrt{\frac{1 \mp v}{1 \pm v}} x^{+}, \quad x^{i^{\prime}}=\lambda x^{i} .
$$

\footnotetext{
${ }^{8}$ Although this is slightly misleading, since we are dealing with a momentum density, which cannot be written as a four vector.
} 
The transformed energy-momentum tensor has one non-vanishing component:

$$
<T_{+^{\prime}+^{\prime}}>=\lambda^{-2} \frac{1+v}{1-v}<T_{++}>\text {. }
$$

Clearly it is not invariant. However, neither is the flat boundary metric:

$$
d s^{2}=\lambda^{-2}\left(d x^{+^{\prime}} d x^{-^{\prime}}+d x^{i^{\prime}} d x^{i^{\prime}}\right)
$$

so to bring the metric back to the original form we must do a Weyl transformation $g_{i j} \rightarrow \lambda^{2} g_{i j}$. The Weyl transformation of the energy-momentum tensor is

$$
<\bar{T}_{+^{\prime}+^{\prime}}>=\lambda^{-(d-2)}<T_{+^{\prime}+^{\prime}}>=\lambda^{-d} \frac{1+v}{1-v}<T_{++}>.
$$

The energy-momentum tensor is therefore invariant under the combined dilatation and boost provided we take $(1+v) /(1-v)=\lambda^{d}$, which gives agreement with equation (5.14).

Note that it is not find any combination of transformations that involves special conformal transformations and leaves the energy-momentum tensor invariant.

\subsection{Scalar 2-point function}

It is well-known that conformal symmetry determines the 2-point functions of operators up to an overall constant?:

$$
\left\langle\mathcal{O}(x) \mathcal{O}\left(x^{\prime}\right)\right\rangle=\frac{c_{\Delta}}{\left(x-x^{\prime}\right)^{2 \Delta}}
$$

where $\Delta$ is the scaling dimension of $\mathcal{O}$. It is not surprising that AdS/CFT reproduces such 2-point functions since they are entirely determined by symmetry, and AdS does indeed give the correct conformal symmetry group.

We are interested in the case when some of the symmetry of the boundary theory has been broken by a background momentum density. The two point function in this case is less constrained. To see this, write

$$
\left\langle\mathcal{O}\left(x_{1}^{+}, x_{1}^{-}, \mathbf{x}_{1}\right) \mathcal{O}\left(x_{2}^{+}, x_{2}^{-}, \mathbf{x}_{2}\right)\right\rangle=f\left(x_{12}^{+}, x_{12}^{-}, \mathbf{x}_{12}^{2}\right),
$$

where we have used the notation $\mathbf{x}$ for $x^{i}$, and $x_{12}$ denotes $x_{1}-x_{2}$. In writing the two point function in this form, we have made use of the unbroken translation and transverse rotation symmetries. Now consider the unbroken symmetries generated by $L_{y}$. Write $\mathbf{x}=(y, z, \ldots)$, and perform the transformation (5.7) with $\lambda=-\sqrt{2} y / x^{+}$. Invariance of $f$ gives

$$
f\left(x^{+}, x^{-}, y^{2}+z^{2}+\ldots\right)=f\left(x^{+}, x^{-}+y^{2} / x^{+}, z^{2}+\ldots\right)
$$

and repeating the same procedure with $L_{z}$, etc. eventually gives

$$
f\left(x^{+}, x^{-}, \mathbf{x}^{2}\right)=f\left(x^{+}, x^{-}+\mathbf{x}^{2} / x^{+}, 1\right) .
$$

So the unbroken Poincaré symmetry has reduced the 2-point function to a function of 2 variables, which can be taken to be $x^{+}$and $x^{2}=x^{+} x^{-}+\mathrm{x}^{2}$. Let us denote this function by $f\left(x^{+}, x^{2}\right)$. Now consider the final unbroken symmetry, generated by $J$. If we assume that $\mathcal{O}$ has scaling dimension $\Delta$ then under the dilatation plus boost symmetry, $\mathcal{O}$ transforms as

$$
\mathcal{O}\left(x^{\prime}\right)=\lambda^{-\Delta} \mathcal{O}(x)
$$

\footnotetext{
${ }^{9}$ Since we are dealing with Lorentzian theories, an $i \epsilon$ term is required, but suppressed throughout this paper.
} 
For the 2-point function, this implies that

$$
f\left(\lambda^{-(d-2) / 2} x^{+}, \lambda^{2} x^{2}\right)=\lambda^{-2 \Delta} f\left(x^{+}, x^{2}\right),
$$

so choosing $\lambda^{2}=1 /\left|x^{2}\right|$ gives

$$
f\left(x^{+}, x^{2}\right)=\frac{1}{\left|x^{2}\right|^{\Delta}} f\left(x^{+}\left|x^{2}\right|^{(d-2) / 4}, \operatorname{sign}\left(x^{2}\right)\right) .
$$

Hence the unbroken symmetries of the boundary theory restrict the 2-point function to take the form

$$
\left\langle\mathcal{O}\left(x_{1}^{+}, x_{1}^{-}, \mathbf{x}_{1}\right) \mathcal{O}\left(x_{2}^{+}, x_{2}^{-}, \mathbf{x}_{2}\right)\right\rangle=\frac{1}{\left|x_{12}^{2}\right|^{\Delta}} f_{ \pm}\left(x_{12}^{+}\left|x_{12}^{2}\right|^{(d-2) / 4}\right),
$$

where $\pm=\operatorname{sign}\left(x^{2}\right)$. The argument of $f_{ \pm}$is invariant under all the unbroken symmetries. In contrast with the conformally invariant case, the functional dependence of the 2-point function is not completely determined by symmetry. The AdS/CFT calculation of the 2-point function therefore has dynamical content for the Kaigorodov spacetime, in contrast with the analogous calculation for pure AdS.

\subsection{AdS/CFT calculation of scalar 2-point function}

Having established that the scalar 2-point function is non-trivial, we shall now calculate it using the AdS/CFT correspondence. The method is similar to that of ([37]) with the exception that we can no longer Wick rotate to Euclidean signature since our the Kaigorodov spacetime is not static. Lorentzian calculations for pure AdS were discussed in [34, 38]. We shall be careful to examine the behaviour of surface terms that arise at the singularity.

Consider a scalar field of mass $\mathrm{DT}^{2}$ :

$$
S_{0}=\int d^{d+1} x \sqrt{-g}\left(-\frac{1}{2}(\partial \phi)^{2}-\frac{1}{2} m^{2} \phi^{2}\right) .
$$

The equation of motion is

$$
\left(\nabla^{2}-m^{2}\right) \phi=0 .
$$

To compute the boundary 2-point function, we have to evaluate the on-shell action of the scalar field solution $\phi(z, x)$ obeying

$$
\phi(\epsilon, x)=\epsilon^{d-\Delta} \phi_{0}(x),
$$

where we have introduced a UV cut-off at $z=\epsilon . \Delta$ is the scaling dimension of the operator $\mathcal{O}$, related to the mass of $\phi$ by [12, 13]

$$
\Delta=\frac{d}{2}+\frac{1}{2} \sqrt{d^{2}+4 m^{2}}
$$

The action can be integrated by parts and the equation of motion used, giving

$$
S_{0}=\sum \int d^{d} x \sqrt{-h}\left(-\frac{1}{2} \phi n \cdot \partial \phi\right)
$$

where the sum runs over the two boundaries $(z=\epsilon$ and $z \rightarrow \infty)$. On each boundary, we have outward unit normal $n=\mp(1 / z) d z$ and the determinant of the boundary metric is $(1 / z)^{d}$.

We shall solve for $\phi$ by Fourier analyzing the $x$ coordinates:

$$
\phi(z, x)=\tilde{\phi}_{k}(z) e^{i k \cdot x},
$$

\footnotetext{
${ }^{10}$ We shall use units for which $l=1$.
} 
where $k \cdot x=\frac{1}{2}\left(k^{+} x^{-}+k^{-} x^{+}\right)+k^{i} x^{i}$. This gives

$$
z^{d-1} \frac{d}{d z}\left(\frac{1}{z^{d-1}} \frac{d \tilde{\phi}_{k}}{d z}\right)-\left(k^{2}+\frac{m^{2}}{z^{2}}-\left(k^{+}\right)^{2} \mu^{d} z^{d}\right) \tilde{\phi}_{k}=0 .
$$

Note that when $k^{+}=0$, this equation is exactly the same as one would obtain in pure AdS, with the solution [37, 38]

$$
\tilde{\phi}_{k}(z)=\epsilon^{d-\Delta} \frac{z^{d / 2} K_{\nu}\left(\sqrt{k^{2}} z\right)}{\epsilon^{d / 2} K_{\nu}\left(\sqrt{k^{2}} \epsilon\right)} \tilde{\phi}_{0}(k),
$$

where $\tilde{\phi}_{0}(k)$ denotes the Fourier transform of $\phi_{0}(x)$. This solution is uniquely determined since the second linearly independent solution gives rise to an infinite surface term at $z=\infty$ [37, 38].

Now consider the case $k^{+} \neq 0$. For small $z$ (i.e. near infinity), neglecting the $z^{d}$ term gives solutions in terms of Bessel functions. For $k^{2}>0$, one obtains

$$
\tilde{\phi}_{k}(z)=\epsilon^{d-\Delta} \frac{z^{d / 2}}{\epsilon^{d / 2}}\left(\frac{I_{-\nu}\left(\sqrt{k^{2}} z\right)+A(k) I_{\nu}\left(\sqrt{k^{2}} z\right)}{I_{-\nu}\left(\sqrt{k^{2}} \epsilon\right)+A(k) I_{\nu}\left(\sqrt{k^{2}} \epsilon\right)}\right) \tilde{\phi}_{0}(k),
$$

where $A(k)$ is some undetermined function and

$$
\nu=\frac{1}{2} \sqrt{d^{2}+4 m^{2}}
$$

which we assume is real, corresponding to the Breitenlohner-Freedman bound [39] $m^{2}>-d^{2} / 4$. For $k^{2}<0$, one obtains

$$
\tilde{\phi}_{k}(z)=\epsilon^{d-\Delta} \frac{z^{d / 2}}{\epsilon^{d / 2}}\left(\frac{J_{-\nu}\left(\sqrt{-k^{2}} z\right)+A(k) J_{\nu}\left(\sqrt{-k^{2}} z\right)}{J_{-\nu}\left(\sqrt{-k^{2}} \epsilon\right)+A(k) J_{\nu}\left(\sqrt{-k^{2}} \epsilon\right)}\right) \tilde{\phi}_{0}(k) .
$$

For large $z$ (i.e. near the singularity), one can neglect the $k^{2}$ and $m^{2} / z^{2}$ terms and obtain the solutions

$$
\tilde{\phi}_{k}=z^{d / 2} J_{ \pm \nu^{\prime}}\left(\frac{2\left|k^{+}\right| \mu^{d / 2}}{d+2} z^{(d+2) / 2}\right)
$$

where

$$
\nu^{\prime}=\frac{d}{d+2}
$$

Near the singularity, the solutions behave as

$$
\tilde{\phi}_{k} \sim z^{(d-2) / 4}\left\{\begin{array}{l}
\sin \\
\cos
\end{array}\right\}\left(z^{(d+2) / 2}\right),
$$

This implies that the surface term near the singularity is bounded but oscillatory as $z \rightarrow \infty$. This is exactly what happens as $z \rightarrow \infty$ for pure AdS when $k^{2}<0$ [38], and can be dealt with by introducing an infra-red cut-off. The two solutions are equally acceptable near the singularity. In contrast with

\footnotetext{
${ }^{11}$ In fact, the equation can also be solved exactly if one retains the $m^{2} / z^{2}$ term and neglects only the $k^{2}$ term, giving the same solution except that $\nu^{\prime}=\sqrt{d^{2}+4 m^{2}} /(d+2)$.
} 
the behaviour in pure AdS, it not possible to uniquely determine the solutions with $k^{2}>0$ by the requirement of finiteness of the surface term at $z=\infty$.

The interpretation of the arbitrary function $A(k)$ was explained in 38]. This function corresponds to the freedom to choose different Lorentzian propagators in the dual CFT. For example, one usually works with the Lorentzian Feynman propagator obtained by analytic continuation of a unique bounded Euclidean propagator. However, there are different propagators (for example the retarded propagator) which one might instead choose to work with. The choice of propagator is determined by boundary conditions, and the freedom to choose boundary conditions is encoded in the function $A(k)$. There may also be freedom in the choice of vacuum state for the CFT.

In all cases, we have written the behaviour for small $z$ as

$$
\tilde{\phi}_{k}(z)=\epsilon^{d-\Delta} K(k, \epsilon, z) \tilde{\phi}_{0}(k),
$$

where

$$
K(k, \epsilon, z)=\frac{\tilde{\phi}_{k}^{-}(z)+A(k) \tilde{\phi}_{k}^{+}(z)}{\tilde{\phi}_{k}^{-}(\epsilon)+A(k) \tilde{\phi}_{k}^{+}(\epsilon)},
$$

and $\tilde{\phi}^{+}$and $\tilde{\phi}^{-}$are the normalizable and non-normalizable [34, 38] solutions, with the behaviour

$$
\tilde{\phi}^{+} \sim z^{\Delta}, \quad \tilde{\phi}^{-} \sim z^{d-\Delta},
$$

as $z \rightarrow 0$. Note that $A(k)$ is not arbitrary when $k^{+}=0$. Evaluating the surface term at $z=\epsilon$ gives 38

$$
S_{0}=\frac{1}{2} \int_{z=\epsilon} d^{d} k d^{d} k^{\prime} \delta^{d}\left(k+k^{\prime}\right) \epsilon^{1-d} \tilde{\phi}_{0}(k) \partial_{z} K(k, \epsilon, z) \tilde{\phi}_{0}(k),
$$

from which one can read off the 2-point function [38]

$$
\left\langle\mathcal{O}(k) \mathcal{O}\left(k^{\prime}\right)\right\rangle=\delta^{d}\left(k+k^{\prime}\right) \lim _{\epsilon \rightarrow 0} \lim _{z \rightarrow \epsilon} \partial_{z} K(k, \epsilon, z) .
$$

At first sight, it appears that this 2-point function will be entirely determined by the part of $K$ coming from the non-normalizable solutions, since these terms dominate as $\epsilon \rightarrow 0$. However, in pure AdS, this part of $K$ turns out to involve only positive integer powers of $k^{2}$. After Fourier transforming back to position space, this gives only contact terms (terms that vanish at non-zero separation, e.g. derivative of delta functions). The non-trivial part of the 2-point function arises from mixing between the normalizable and non-normalizable solutions, which gives non-integer powers of $k^{2}$ [37, 38].

For the Kaigorodov spacetime, we have to be a bit more careful since we know that $\tilde{\phi}^{ \pm}$will be corrected from their pure AdS forms by subleading terms that depend on $\mu$. These subleading terms could dominate the terms arising from mixing between the normalizable and non-normalizable solutions, and therefore change the form of the 2-point function. We can examine this $\mu$ dependence by seeking series solutions for $\tilde{\phi}^{ \pm}$near $z=0$. One obtains $[2]$ :

$$
\begin{gathered}
\tilde{\phi}_{k}^{-}=z^{d-\Delta}\left(a_{0}+a_{2} z^{2}+\ldots\right), \\
\tilde{\phi}_{k}^{+}=z^{\Delta}\left(b_{0}+b_{2} z^{2}+\ldots\right),
\end{gathered}
$$

with the series agreeing with the approximate Bessel function solutions up to $d$-th order, but then being altered by the $\mu$ dependence. The deviation from the Bessel function solutions at $(d+2)$-th order is

$$
\delta a_{d+2}=\frac{\mu^{d} k^{+2}}{2(\Delta-d-1)(d+2)} a_{0}, \quad \delta b_{d+2}=-\frac{\mu^{d} k^{+2}}{2(\Delta+1)(d+2)} b_{0} .
$$

\footnotetext{
${ }^{12}$ If $\nu$ is an integer then there will be logarithmic terms in these series. We shall concentrate on the case of non-integral $\nu$ for simplicity.
} 
Since the terms $a_{0}, \ldots a_{d}, b_{0} \ldots b_{d}$ and the Bessel function parts of $a_{d+2}$ and $b_{d+2}$ only involve positive integer powers of $k^{2}$, it follows that $a_{d+2}$ and $b_{d+2}$ only involve positive integer powers of $k^{2}$ and $k^{+}$, which means that they will still only give contact terms in the 2-point function. Thus the nontrivial contribution to the 2-point function will come from the leading order terms in $\tilde{\phi}^{ \pm}$, which are independent of $\mu$. It follows that the 2-point function will not depend on $\mu$.

The only novel feature that arises from this calculation is that the 2-point function is uniquely determined in momentum space when $k^{+}=0$, but not when $k^{+} \neq 0$. This is to be contrasted with the calculation for pure AdS, which gives a unique propagator when $k^{2}>0$ but not when $k^{2}<0$, which is what one expects in field theory [3]. The occurence of $k^{+}$in our results is characteristic of the infinite momentum frame. The freedom in the propagator when $k^{+} \neq 0$ and $k^{2}>0$ is presumably related to some subtlety of quantization in the infinite momentum frame.

Since there is no dependence on $\mu$, we are free to choose the function $A(k)$ so that the 2-point function that we obtain from the Kaigorodov spacetime is the same as the 2-point function that we obtain from pure AdS by Wick rotation from Euclidean signature. The only thing we have to worry about it whether this gives the correct result when $k^{+}=0$, which it must do since the bulk solution is the same for Kaigorodov and for pure AdS in this case. We therefore obtain the Feynman propagator:

$$
\left\langle\mathcal{O}(x) \mathcal{O}\left(x^{\prime}\right)\right\rangle=\frac{c_{\Delta}}{\left(\left(x-x^{\prime}\right)^{2}-i \epsilon\right)^{\Delta}},
$$

where $c_{\Delta}$ is given in [37].

\subsection{Attempted explanation}

The simplicity of the AdS/CFT result suggests that there should be some way of understanding it in purely field-theoretic terms. The best we have come up with is the following.

Writing the function $f$ of (5.27) in terms of a dimensionless variable, one obtains

$$
f\left(P^{1 / 2} x^{+}\left(x^{2}\right)^{(d+2) / 4}, N, \lambda\right)
$$

where we have now included the dependence on $N$ and $\lambda$ explicitly. Our AdS/CFT calculation is valid for $N, \lambda, P \rightarrow \infty$ with $\mu^{d} \sim P / N^{d / 2}$ fixed. We would like to argue that $f$ must approach a constant in this limit, namely $f(\infty, \infty, \infty)$. This would explain our AdS/CFT result. However, this argument would break down if, at large $N, f$ reduced to a function of

$$
N^{-d / 4} P^{1 / 2} x^{+}\left(x^{2}\right)^{(d-2) / 4} \sim \mu^{d / 2} x^{+}\left(x^{2}\right)^{(d-2) / 4} .
$$

In order to improve on this argument, one would need to understand the explicit $N$-dependence of the 2-point function, at least for large $N$. If $P$ were kept finite in the large $N$ limit then perhaps it would be possible to extend the usual planar-graph arguments (see e.g. [40]) of large $N$ field theory to fix the $N$-dependence of $f$. However, when $P$ scales with $N$, one is dealing with field theory in an $N$-dependent background, which is probably much harder to understand.

\footnotetext{
${ }^{13}$ We remind the reader why this is so. In free field theory, the momentum space propagator is obtained from the equation $\left(k^{2}+m^{2}\right) G\left(k ; m^{2}\right)=1$. The solution is uniquely defined except when $k^{2}=-m^{2}$, corresponding to the freedom to add to $G\left(k ; m^{2}\right)$ a function of the form $g(k) \delta\left(k^{2}+m^{2}\right)$. In the interacting case, one can write the 2-point function in a Källen-Lehmann representation as an integral over free field propagators weighted by some spectral function $\sigma\left(m^{2}\right)$. This spectral function vanishes for $m^{2}<0$, guaranteeing that the arbitrariness in the free field propagator does not affect the interacting propagator when $k^{2}>0$.
} 


\section{Discussion}

We have discussed spacetimes describing pp-waves propagating on the worldvolume of extremal nondilatonic branes. We have explicitly shown that any inertial observer in the bulk of such a spacetime will encounter infinite tidal forces at the place where the horizon would ordinarily be located. This means that these solutions cannot be extended through the horizon region, so the spacetime terminates at a null singularity. This is evidence in support of the idea [2, 3] that there may be a generalized "no-hair" theorem that deals with time-dependent "hair".

In the near-horizon limit, these spacetimes reduce to spacetimes describing pp-waves moving along the horospheres of AdS. We have discussed an apparent Virasoro symmetry [15] of such spacetimes, and shown that it arises from an action of the Virasoro group on three dimensional asymptotically AdS submanifolds. It would be interesting to see whether this symmetry could be used to extend the entropy calculation of [41] to extended objects in higher dimensional AdS, for example the BTZ black string of [42].

We have shown that pp-waves moving tangential to the horospheres of AdS will make the AdS horizon become singular unless the wave does not extend as far as this horizon. This result has implications for the Randall-Sundrum [21] scenario in which our universe is regarded as a thin domain wall in AdS. This thin domain wall should presumably be regarded as a mathematical idealization of a thick domain wall in AdS. In [22], it was shown that the pp-waves propagating tangentially to such a domain wall correspond precisely to the gravitons of the Randall-Sundrum scenario. We have shown that any of these pp-waves will induce a curvature singularity at the AdS horizon. However, this horizon is the surface at which one must impose boundary conditions for the bulk spacetime. Thus one is faced with the unappealing prospect of having to impose boundary conditions at a naked singularity, which presumably requires an understanding of quantum gravity. If one were to impose the boundary condition that the past horizon remain regular then it appears that one must exclude the possibility of there being gravitons present on the domain wall!

We have presented evidence in favour of the conjecture [9] that gravity in the Kaigorodov spacetime is dual to a CFT in the infinite momentum frame with constant background momentum density. In particular, we have calculated the CFT energy momentum tensor that arises from a large class of asymptotically AdS pp-waves, including $K_{d+1}$. We have discussed how the isometries of $K_{d+1}$ have a natural interpretation as the subgroup of the conformal group that leaves the background momentum density invariant. This symmetry group determines the 2-point function of a scalar operator only up to an arbitrary function of one variable. Our AdS/CFT calculation of the 2-point function therefore has dynamical content, and is found to agree with the result one obtains from pure AdS (i.e., when the conformal symmetry group is unbroken). We have suggested that this is a large $N$ effect. The 2-point function exhibits some ambiguity associated with working in Lorentzian signature, however the ambiguity does not take the expected form. This is perhaps the only signal in our work that the boundary CFT has really been quantized in the infinite momentum frame.

It might be possible to understand our results more clearly by doing a free field theory calculation in the CFT. The quantum state of the CFT could be constructed by taking a thermal density matrix (corresponding to a non-extremal stack of branes), applying a finite boost, and then taking the limit in which the temperature tends to zero and the boost to infinity with the energy density fixed.

It would be interesting to have a complete understanding of how string theory resolves the Kaigorodov singularity. Certainly, if we use the criterion of Gubser [43] then this singularity is "good", in the sense that the slightest finite temperature deformation will yield a non-extremal brane-wave, which we know is simply a boosted non-extremal brane and therefore has a regular horizon [9]. On the other hand, it would be nice to know if the singularity in the bulk could be explicitly resolved along the lines of recent work [44, 45] which deals primarily with timelike singularities. 
We should also mention that our discussion of the Kaigorodov metric has been confined to the case $\mu^{d}>0$. However, the metric with $\mu^{d}<0$ is still a solution of Einstein's equations. This metric gives a CFT energy-momentum tensor with negative energy, which is unacceptable. So it appears that AdS/CFT is telling us that the $\mu^{d}<0$ Kaigorodov singularity cannot be resolved. Further support for this idea comes from the fact that the metric in this case does not have a regular finite temperature deformation.

As we have seen, it is easy to write down pp-wave solutions which are asymptotic to AdS and which induce non-constant null momentum density in the boundary theory. For these spacetimes, we would expect a non-trivial 2-point function in the CFT. It would be interesting to investigate such spacetimes in more detail. A particular case of interest would be to choose $F\left(x^{+}, z\right)=\mu^{d} x^{+p} z^{d}$, with $p$ an integer (to avoid problems when $x^{+}<0$ ). Such a bulk spacetime would admit a scaling symmetry analagous to the Kaigorodov scaling symmetry.

Of course, not all gravitons in AdS will move tangential to the horospheres (and therefore the boundary) of AdS. As discussed in [46, 47], information about a graviton emitted from deep in the bulk of AdS which heads towards the boundary of AdS must be encoded nonlocally in Wilson loops of the boundary CFT, with the size of the Wilson loop related to the distance of the disturbance from the boundary. These degrees of freedom in the CFT which encode information about the graviton in the bulk were dubbed "precursors". An interesting (and open) problem is to find a full non-linear solution describing a graviton moving in the direction transverse to the horospheres, so that we might have a hope of explicitly computing properties of these precursors.

\section{Acknowledgments}

DB would like to thank Simon Ross and Paul Saffin for discussions, and Simon Ross for comments on a draft of this paper. DB is supported in part by the EPSRC grant GR/N34840/01. AC thanks Mirjam Cvetic, Dan Freedman and Andreas Karch for useful conversations. AC is supported in part by funds provided by the U.S. Department of Energy (D.O.E.) under cooperative research agreement DE-FC02-94ER40818. HSR thanks Jerome Gauntlett and Prem Kumar for discussions and is funded by PPARC.

\section{A Appendix}

Our line element is

$$
d s^{2}=H(r)^{-2 / d}\left(2 d x^{+} d x^{-}+F\left(x^{+}, x^{i}, r\right) d x^{+2}+d x^{i} d x^{i}\right)+H(r)^{2 / \tilde{d}}\left(d r^{2}+r^{2} d \Omega_{\tilde{d}+1}^{2}\right) .
$$

We shall compute the curvature tensors in the following basis:

$$
\begin{aligned}
e^{+} & =H^{-1 / d} d x^{+}, \quad e^{-}=H^{-1 / d}\left(d x^{-}+\frac{1}{2} F d x^{+}\right), \quad e^{i}=H^{-1 / d} d x^{i}, \\
e^{r} & =H^{1 / \tilde{d}} d r, \quad e^{m}=H^{1 / \tilde{d}} r \hat{e}^{m},
\end{aligned}
$$

where $\hat{e}^{m}$ are an orthonormal basis of 1 -forms with respect to the metric $\hat{g}_{m n}$ associated with the line element $d \Omega_{\tilde{d}+1}^{2}$. The metric can be written

$$
g_{M N}=\eta_{A B} e^{A}{ }_{M} e^{B}{ }_{N},
$$

where

$$
\begin{aligned}
\eta_{+-}=\eta_{-+} & =\eta_{r r}=1 \\
\eta_{i j} & =\delta_{i j} \\
\eta_{m n} & =\delta_{m n}
\end{aligned}
$$


is the Minkowski metric. Note that we are using letters $M, N, \ldots$ as curved (coordinate) indices and $A, B, \ldots$ as basis indices.

The connection 1-forms are defined by

$$
d e^{A}=-\omega_{B}^{A} \wedge e^{B}
$$

The non-vanishing connection 1-forms are

$$
\begin{gathered}
\omega_{+i}=\omega_{i}^{-}=\frac{1}{2} H^{1 / d} \frac{\partial F}{\partial x^{i}} e^{+}, \\
\omega_{+r}=\omega_{r}^{-}=-\frac{1}{d} H^{-1 / \tilde{d}} \frac{H^{\prime}}{H} e^{-}+\frac{1}{2} H^{-1 / \tilde{d}} \frac{\partial F}{\partial r} e^{+}, \\
\omega_{-r}=\omega_{r}^{+}=-\frac{1}{d} H^{-1 / \tilde{d}} \frac{H^{\prime}}{H} e^{+}, \\
\omega_{i r}=\omega_{r}^{i}=-\frac{1}{d} H^{-1 / \tilde{d}} \frac{H^{\prime}}{H} e^{i}, \\
\omega_{r m}=\omega_{m}^{r}=-H^{-1 / \tilde{d}}\left(\frac{H^{\prime}}{\tilde{d} H}+\frac{1}{r}\right) e^{m}, \\
\omega_{m n}=\hat{\omega}_{m n},
\end{gathered}
$$

where $\hat{\omega}_{m n}$ are the connection 1-forms associated with $\hat{e}^{m}$. Other non-vanishing components are obtained from the ones given above by antisymmetry: $\omega_{A B}=-\omega_{B A}$.

The curvature 2 -form is defined by

$$
\Theta_{A B}=d \omega_{A B}+\omega_{A C} \wedge \omega_{B}^{C}
$$

The non-vanishing curvature 2-forms are

$$
\begin{aligned}
& \Theta_{+-}=\frac{1}{d^{2}} H^{-2 / \tilde{d}}\left(\frac{H^{\prime}}{H}\right)^{2} e^{+} \wedge e^{-}, \\
& \Theta_{+i}=\frac{1}{2 d} H^{-2 / \tilde{d}} \frac{H^{\prime}}{H} \frac{\partial F}{\partial r} e^{+} \wedge e^{i}-\frac{1}{2} H^{2 / d} \frac{\partial^{2} F}{\partial x^{i} \partial x^{j}} e^{+} \wedge e^{j} \\
& -\frac{1}{2} H^{1 / d-1 / \tilde{d}} \frac{\partial^{2} F}{\partial x^{i} \partial r} e^{+} \wedge e^{r}-\frac{1}{d^{2}} H^{-2 / d t}\left(\frac{H^{\prime}}{H}\right)^{2} e^{-} \wedge e^{i} \\
& \Theta_{+r}=-\frac{1}{2} H^{1 / d-1 / \tilde{d}} \frac{\partial^{2} F}{\partial x^{i} \partial r} e^{+} \wedge e^{i}+\frac{1}{2} H^{-2 / \tilde{d}}\left(-\frac{\partial^{2} F}{\partial r^{2}}+\left(\frac{2}{d}+\frac{1}{\tilde{d}}\right) \frac{H^{\prime}}{H} \frac{\partial F}{\partial r}\right) e^{+} \wedge e^{r} \\
& +\frac{1}{d} H^{-2 / \tilde{d}}\left(\frac{H^{\prime \prime}}{H}-\left(\frac{1}{d}+\frac{1}{\tilde{d}}+1\right)\left(\frac{H^{\prime}}{H}\right)^{2}\right) e^{-} \wedge e^{r}, \\
& \Theta_{+m}=-\frac{1}{2} H^{-2 / \tilde{d}}\left(\frac{H^{\prime}}{\tilde{d} H}+\frac{1}{r}\right) \frac{\partial F}{\partial r} e^{+} \wedge e^{m}+\frac{1}{d} H^{-2 / \tilde{d}} \frac{H^{\prime}}{H}\left(\frac{H^{\prime}}{\tilde{d} H}+\frac{1}{r}\right) e^{-} \wedge e^{m} \\
& \Theta_{-i}=-\frac{1}{d^{2}} H^{-2 / \tilde{d}}\left(\frac{H^{\prime}}{H}\right)^{2} e^{+} \wedge e^{i}
\end{aligned}
$$




$$
\begin{gathered}
\Theta_{-r}=\frac{1}{d} H^{-2 / d}\left(\frac{H^{\prime \prime}}{H}-\left(\frac{1}{d}+\frac{1}{\tilde{d}}+1\right)\left(\frac{H^{\prime}}{H}\right)^{2}\right) e^{+} \wedge e^{r} \\
\Theta_{-m}=\frac{1}{d} H^{-2 / \tilde{d}} \frac{H^{\prime}}{H}\left(\frac{H^{\prime}}{\tilde{d} H}+\frac{1}{r}\right) e^{+} \wedge e^{m} \\
\Theta_{i j}=-\frac{1}{d^{2}} H^{-2 / \tilde{d}}\left(\frac{H^{\prime}}{H}\right)^{2} e^{i} \wedge e^{j} \\
\Theta_{i r}=\frac{1}{d} H^{-2 / \tilde{d}}\left(\frac{H^{\prime \prime}}{H}-\left(\frac{1}{d}+\frac{1}{\tilde{d}}+1\right)\left(\frac{H^{\prime}}{H}\right)^{2}\right) e^{i} \wedge e^{r} \\
\Theta_{i m}=\frac{1}{d} H^{-2 / \tilde{d}} \frac{H^{\prime}}{H}\left(\frac{H^{\prime}}{\tilde{d} H}+\frac{1}{r}\right) e^{i} \wedge e^{m} \\
\Theta_{r m}=-\frac{1}{\tilde{d}} H^{-2 / \tilde{d}}\left(\frac{H^{\prime \prime}}{H}-\left(\frac{H^{\prime}}{H}\right)^{2}+\frac{H^{\prime}}{H r}\right) e^{r} \wedge e^{m} \\
\Theta_{m n}=\hat{\Theta}_{m n}-H^{-2 / \tilde{d}}\left(\frac{H^{\prime}}{\tilde{d} H}+\frac{1}{r}\right)^{2} e^{m} \wedge e^{n},
\end{gathered}
$$

with the other non-vanishing components related to these by antisymmetry. The Riemann curvature tensor is defined by

$$
\Theta_{A B}=\frac{1}{2} R_{A B C D} e^{C} \wedge e^{D} .
$$

The non-zero components are

$$
\begin{gathered}
R_{+-+-}=\frac{1}{d^{2}} H^{-2 / \tilde{d}}\left(\frac{H^{\prime}}{H}\right)^{2}, \\
R_{+i+j}=-\frac{1}{2} H^{2 / d} \frac{\partial^{2} F}{\partial x^{i} \partial x^{j}}+\frac{1}{2 d} H^{-2 / \tilde{d}} \frac{H^{\prime}}{H} F^{\prime} \delta_{i j}, \\
R_{+i+r}=-\frac{1}{2} H^{1 / d-1 / \tilde{d}} \frac{\partial^{2} F}{\partial r \partial x^{i}}, \\
R_{+i-j}=-\frac{1}{d^{2}} H^{-2 / \tilde{d}}\left(\frac{H^{\prime}}{H}\right)^{2} \delta_{i j}, \\
R_{+r+r}=\frac{1}{2} H^{-2 / \tilde{d}}\left(-\frac{\partial^{2} F}{\partial r^{2}}+\left(\frac{2}{d}+\frac{1}{\tilde{d}}\right) \frac{H^{\prime}}{H} \frac{\partial F}{\partial r}\right), \\
R_{+m+n}=\frac{1}{d} H^{-2 / \tilde{d}}\left(\frac{H^{\prime \prime}}{H}-\left(\frac{1}{d}+\frac{1}{\tilde{d}}+1\right)\left(\frac{H^{\prime}}{H}\right)^{2}\right), \\
R_{+m-n}=\frac{1}{d} H^{-2 / \tilde{d}} \frac{H^{\prime}}{H}\left(\frac{H^{\prime}}{\tilde{d} H}+\frac{1}{r}\right) F^{\prime} \delta_{m n}, \\
R_{i j k l}=-\frac{1}{\tilde{d}^{2} H} H^{-2 / \tilde{d}}\left(\frac{H^{\prime}}{H}\right)^{2}\left(\delta_{i k} \delta_{j l}-\delta_{i l} \delta_{j k}\right), \\
R_{i r j r}=\frac{1}{d} H^{-2 / \tilde{d}}\left(\frac{H^{\prime \prime}}{H}-\left(\frac{1}{d}+\frac{1}{\tilde{d}}+1\right)\left(\frac{H^{\prime}}{H}\right)^{2}\right) \delta_{i j},
\end{gathered}
$$




$$
\begin{gathered}
R_{i m j n}=\frac{1}{d} H^{-2 / \tilde{d}} \frac{H^{\prime}}{H}\left(\frac{H^{\prime}}{\tilde{d} H}+\frac{1}{r}\right) \delta_{i j} \delta_{m n}, \\
R_{r m r n}=-\frac{1}{\tilde{d}} H^{-2 / \tilde{d}}\left(\frac{H^{\prime \prime}}{H}-\left(\frac{H^{\prime}}{H}\right)^{2}+\frac{H^{\prime}}{H r}\right) \delta_{m n}, \\
R_{m n p q}=\hat{R}_{m n p q}-H^{-2 / \tilde{d}}\left(\frac{H^{\prime}}{\tilde{d} H}+\frac{1}{r}\right)^{2}\left(\delta_{m p} \delta_{n q}-\delta_{m q} \delta_{n p}\right),
\end{gathered}
$$

Other non-vanishing components are related to these by the symmetries of the Riemann tensor. The Ricci tensor is

$$
R_{A B}=R_{A B C}^{C}=R_{A-B+}+R_{A+B-}+R_{A i B i}+R_{A r B r}+R_{A m B m} .
$$

The non-vanishing components are

$$
R_{++}=-\frac{1}{2} H^{-2 / \tilde{d}}\left(\frac{\partial^{2} F}{\partial r^{2}}+\frac{\tilde{d}+1}{r} \frac{\partial F}{\partial r}\right)-\frac{1}{2} H^{2 / d} \nabla_{\perp}^{2} F,
$$

where $\nabla_{\perp}^{2}$ is the Laplacian with respect to the flat coordinates $x^{i}$,

$$
\begin{gathered}
R_{+-}=\frac{1}{d} H^{-2 / \tilde{d}}\left(\frac{H^{\prime \prime}}{H}-\left(\frac{H^{\prime}}{H}\right)^{2}+\frac{\tilde{d}+1}{r} \frac{H^{\prime}}{H}\right) \\
R_{i j}=\frac{1}{d} H^{-2 / \tilde{d}}\left(\frac{H^{\prime \prime}}{H}-\left(\frac{H^{\prime}}{H}\right)^{2}+\frac{\tilde{d}+1}{r} \frac{H^{\prime}}{H}\right) \delta_{i j}, \\
R_{r r}=-\frac{1}{\tilde{d}} H^{-2 / \tilde{d}}\left(\frac{H^{\prime \prime}}{H}+\frac{\tilde{d}}{d}\left(\frac{H^{\prime}}{H}\right)^{2}+\frac{\tilde{d}+1}{r} \frac{H^{\prime}}{H}\right), \\
R_{m n}=\hat{R}_{m n}-\frac{1}{\tilde{d}} H^{-2 / \tilde{d}}\left(\frac{H^{\prime \prime}}{H}-\left(\frac{H^{\prime}}{H}\right)^{2}+\frac{\tilde{d}+1}{r} \frac{H^{\prime}}{H}+\frac{1}{r^{2}}\right) \delta_{m n} \\
=-\frac{1}{\tilde{d}} H^{-2 / \tilde{d}}\left(\frac{H^{\prime \prime}}{H}-\left(\frac{H^{\prime}}{H}\right)^{2}+\frac{\tilde{d}+1}{r} \frac{H^{\prime}}{H}\right) \delta_{m n},
\end{gathered}
$$

where in the final expression we have used the fact that the 'internal' space is an Einstein space, which in our basis implies

$$
\hat{R}_{m n}=\frac{\tilde{d}}{r^{2}} H^{-2 / \tilde{d}} \delta_{m n}
$$

\section{References}

[1] G. W. Gibbons and P. K. Townsend. Phys. Rev. Lett., 71:3754, 1993. hep-th/9302049.

[2] N. Kaloper, R. C. Myers, and H. Roussel. Phys. Rev., D55:7625, 1997. hep-th/9612248.

[3] G. T. Horowitz and H. Yang. Phys. Rev., D55:7618, 1997. hep-th/9701077.

[4] R. C. Myers. Gen. Rel. Grav., 29:1217, 1997. gr-qc/9705065.

[5] S. Ross. JHEP, 9808:003, 1998. hep-th/9710158. 
[6] H. Yang. Localized Intersecting Brane Solutions of D=11 Supergravity, 1999. hep-th/9902128.

[7] D. Youm. Localized Intersecting BPS Branes, 1999. hep-th/9902208.

[8] J. Maldacena. Adv. Theor. Math. Phys., 2:231, 1998. hep-th/9711200.

[9] M. Cvetic, H. Lü, and C. N. Pope. Nucl. Phys., B545:309, 1999. hep-th/9810123.

[10] V. R. Kaigorodov. Dokl. Akad. Nauk. SSSR, 146:793, 1962.

[11] V. R. Kaigorodov. Sov. Phys. Doklady, 7:893, 1963.

[12] S. S. Gubser, I. R. Klebanov and A. M. Polyakov. Phys. Lett., B428:105, 1998. hep-th/9802109.

[13] E. Witten. Adv. Theor. Math. Phys., 2:253, 1998. hep-th/9802150.

[14] J. D. Brown and M. Henneaux. Commun, Math. Phys., 104:207, 1986.

[15] M. Bañados, A. Chamblin, and G. W. Gibbons. Phys. Rev., D61:081901, 2000. hep-th/9911101.

[16] R. G. Russo and A. A. Tesytlin. Nucl. Phys., B490:121, 1997. hep-th/9611047.

[17] D. Brecher and M. J. Perry. Nucl. Phys., B566:151, 2000. hep-th/9908018.

[18] B. Janssen. JHEP, 0001:044, 2000. hep-th/9910077.

[19] J. M. Figueroa-O'Farrill. Phys. Lett., B471:128, 1999. hep-th/9910086.

[20] J. Podolský. Class. Quant. Grav., 15:719, 1998. gr-qc/9801052.

[21] L. Randall and R. Sundrum. Phys. Rev. Lett., 83:4690, 1999. hep-th/9906064.

[22] A. Chamblin and G. W. Gibbons. Phys. Rev. Lett., 84:1090, 1999. hep-th/9909130.

[23] M. Bañados, C. Teitelboim, and J. Zanelli, Phys. Rev. Lett., 69:1849, 1992. hep-th/9204099.

[24] M. Bañados, M. Henneaux, C. Teitelboim, and J. Zanelli. Phys. Rev., D48:1506, 1993. grqc/9302012.

[25] K. Behrndt and D. Lüst. JHEP, 9907:019, 1999. hep-th/9905180.

[26] H. Lü and C. N. Pope and P. K. Townsend. Phys. Lett., B391:39, 1997. hep-th/9607164.

[27] M. Henningson and K. Skenderis. JHEP, 9807:023, 1998. hep-th/9806087.

[28] A. Tseytlin and H. Liu. Nucl. Phys., B533:88, 1998. hep-th/9804083.

[29] M. Henningson and K. Skenderis. Fortsch. Phys., 48:125, 2000. hep-th/9812032.

[30] V. Balasubramanian and P. Kraus. Commun. Math. Phys., 208:413, 1999. hep-th/9902121.

[31] R. Emparan, C. V. Johnson and R. C. Myers. Phys. Rev., D60:104001, 1999. hep-th/9903238.

[32] P. Kraus, F. Larsen, and R. Siebelink. Nucl. Phys., B563:259, 1999. hep-th/9906127.

[33] R. M. Wald. General Relativity. The University of Chicago Press, 1984.

[34] V. Balasubramanian, P. Kraus and A. Lawrence. Phys. Rev., D59:046003,1999. hep-th/9805171. 
[35] J. Maldacena and A. Strominger. JHEP, 9812:005, 1998. hep-th/9804085.

[36] J. Polchinski. String Theory. Cambridge University Press, 1998.

[37] D. Z. Freedman, S. D. Mathur, A. Matusis and L. Rastelli. Nucl. Phys., B546:96, 1999. hepth/9804058.

[38] V. Balasubramanian, P. Kraus, A. Lawrence and S. P. Trivedi. Phys. Rev., D59:104021, 1999. hep-th/9808017.

[39] P. Breitenlohner and D. Z. Freedman. Ann. Phys., 144:249, 1982.

[40] A. V. Manohar. Large N QCD, (1997 Les Houches lectures). hep-ph/9802419.

[41] A. Strominger. JHEP, 9802:009, 1998. hep-th/9712251.

[42] R. Emparan, G. T. Horowitz and R. C. Myers. JHEP, 0001:021, 2000. hep-th/9912135.

[43] S. S. Gubser. Curvature Singularities: the Good, the Bad, and the Naked, 2000. hep-th/0002160.

[44] C. V. Johnson, A. W. Peet, J. Polchinski. Phys. Rev. D61:086001, 2000. hep-th/9911161.

[45] J. Polchinski, M. Strassler. The String Dual of a Confining Four-Dimensional Gauge Theory, 2000. hep-th/0003136.

[46] J. Polchinski, L. Susskind and N. Toumbas. Phys.Rev., D60:084006, 1999. hep-th/9903228

[47] L. Susskind and N. Toumbas. Phys. Rev. D61:044001, 2000. hep-th/9909013. 